QMW-95-50

hep-th/9512181

December 1995

\title{
STRING DYNAMICS AT STRONG COUPLING
}

\author{
C.M. HulL \\ Physics Department, Queen Mary and Westfield College, \\ Mile End Road, London E1 4NS, U.K.
}

\begin{abstract}
The dynamics of superstring, supergravity and $M$ theories and their compactifications are probed by studying the various perturbation theories that emerge in the strong and weak coupling limits for various directions in coupling constant space. The results support the picture of an underlying non-perturbative theory that, when expanded perturbatively in different coupling constants, gives different perturbation theories, which can be perturbative superstring theories or superparticle theories. The $p$-brane spectrum is considered in detail and a criterion found to establish which $p$-branes govern the strong coupling dynamics. In many cases there are competing conjectures in the literature, and this analysis decides between them. In other cases, new results are found. The chiral six-dimensional theory resulting from compactifying the type IIB string on $K_{3}$ is studied in detail and it is found that certain strong coupling limits appear to give new theories, some of which hint at the possibility of a twelve-dimensional origin.
\end{abstract}




\section{Introduction}

String theory is defined perturbatively through a set of rules for calculating scattering amplitudes. However, recent progress has led to some striking conjectures regarding the non-perturbative structure of the theory that have passed many tests [1-26]. The picture that seems to be emerging is that there is some as yet unknown theory that, when expanded perturbatively, looks like a perturbative string theory, but which has a surprisingly simple structure at the non-perturbative level which includes $\mathrm{U}$ or S duality symmetries relating perturbative states to solitons, and weak coupling to strong. Moreover, there are a number of different coupling constants corresponding to the expectation values of various scalars, and the perturbation expansions with respect to some of these define string theories, but different string theories arise for different coupling constants. This leads to unexpected equivalences between string theories that look very different in perturbation theory: they result from different perturbation expansions of the same theory. In many cases, the strong coupling limit of a given theory with respect to a particular coupling constant is described by the weak coupling expansion of a dual theory, which is sometimes another string theory and sometimes a field theory.

An example which illustrates many of these points is the one obtained from the toroidal compactification of the heterotic string to four dimensions on $T^{6}$. When the full non-perturbative theory, including solitons, is considered, there is strong evidence that the theory has an $S L(2, \mathbb{Z})$ S-duality symmetry relating strong to weak coupling and interchanging electric and magnetic charges $[11,12,13]$. The theory is then self-dual: the strong coupling limit is described by the weak-coupling expansion of a dual heterotic string theory, which is of exactly the same form, but with magnetic charges arising in the perturbative spectrum while electric ones arise as solitons. Expanding the same theory in other directions in coupling constant space can give the perturbative expansion of the type IIA string or of the type IIB string compactified on $K_{3} \times T^{2}$ [1], leading to the conjectured equivalence of the type II and heterotic strings. The expansion with respect to other coupling 
constants of the theory has been considered in [5].

The $p$-brane states of the theory play a crucial role in understanding the nonperturbative structure [1]. These are associated with $p$-brane solutions of the effective low-energy supergravity theory that saturate a Bogomolnyi bound. As some of these solutions are singular, the question arises as to whether they should be associated with states in the quantum spectrum. For example, type II superstring theories in ten dimensions have a string and a 5-brane coupling to the 2 -form in the NS-NS (Neveu-Schwarz/Neveu-Schwarz) sector together with various $p$-branes coupling to the antisymmetric tensor gauge fields in the RR (Ramond-Ramond) sector [28,31]. The 5-brane is non-singular and can be regarded as a soliton of weakly-coupled string theory [29,30]. The NS-NS 1-brane solution [27] is singular, but should be regarded as the field configuration outside a fundamental string source. Many of the RR $p$-brane solutions are singular, but can each be regarded as the field configuration outside a D-brane source [23]. The NS-NS solitons arise as conventional conformally invariant sigma-models, while the RR branes arise as D-branes [23]. Alternatively, the singular $p$-brane solutions of the IIA theory have a non-singular origin in the 11-dimensional theory, so that the 11-dimensional picture allows all $p$-branes to be included in the theory [4].

However, although an attractive picture is emerging, there remain many open questions. For example, the strong coupling limit of the $S O(32)$ heterotic string in 10 dimensions has been variously conjectured to be a five-brane theory $[32,30]$ or a type I superstring theory [3,33] or 11-dimensional supergravity compactified on a one-dimensional degeneration of $K_{3}$ [5], and the question arises as to which, if any, of these is correct. The first two appear at first to be on a very similar footing. A change of variables in the heterotic string low-energy effective action gives the low-energy effective action of the type I string [3] while a different change of variables gives a supergravity theory with a 6-form gauge potential (instead of a 2-form potential) that has been proposed as the low-energy effective action of some as yet unknown five-brane theory [32,30], which might bear a similar relation to the 6-form version of the supergravity theory as M-theory does to 11-dimensional 
supergravity. In each case, the change of variables includes a change in the sign of the dilaton, so that the strong coupling limit of the heterotic string could agree with the weak coupling limit of either the type I string or five-brane. In $[9,10,25]$ it was argued that the heterotic string arises as a soliton of the type I theory, but it is straightforward to see that it also emerges as a soliton of the proposed five-brane effective action. String/five-brane duality in $D=10$ is supported by its relation to string/string duality in $D=6$ [8] (further evidence for which is proposed in $[6,7]$ ), while the heterotic/type I duality is supported by its relation to the $S L(2, \mathbb{Z})$ duality of the type IIB string [9], and the evidence presented in [25]. So how are we to choose between these two conjectures, and how are they related to that of [5]?

Another issue is that of the strong coupling limit of the type IIA theory. Similar arguments to those used in the heterotic string suggest that this might be a type II five-brane theory, while those of [2,3] suggest an 11-dimensional supergravity, supermembrane or $\mathrm{M}$ theory. In [3] it was argued that the 0-brane solitons or extreme black holes of the type IIA theory in $D=10$ have masses that scale as $g^{-1}$ where $g$ is the string coupling constant. These become massless in the strong coupling limit and can be identified with the Kaluza-Klein modes of 11dimensional supergravity compactified on a circle of radius $R \sim g^{2 / 3}$ [3], leading to the conjecture that the strong coupling limit is 11-dimensional supergravity. These 'extreme black holes' are in fact singular solutions of the $D=10$ supergravity theory, but can be associated with $p=0$ D-branes. Moreover, the theory also has RR $p$-branes with $p=2,4,6$ (and $8[23,34]$ ) whose mass per unit $p$-volume also scales as $g^{-1}$, together with a NS-NS five-brane whose density scales as $g^{-2}$ so that these all appear to become 'massless' in the strong coupling limit. More properly, they appear to become null $p$-branes with vanishing density and zero tension whose world-volume is a null $(p+1)$-surface. These extra massless solitons could spoil the interpretation as an 11-dimensional field theory; indeed, the fact that the fivebrane appears to become massless faster than the other $p$-branes might be taken as evidence in favour of a dual five-brane theory. Even if the 11-dimensional conjecture 
is accepted, there remains the question as to whether the strong coupling limit is a supergravity theory as suggested in [3] or whether it is some supermembrane or M theory, since the analysis of [3] only addresses a particular class of particle states.

Similar remarks apply to the type IIB theory, which again could be dual to a type IIB 5-brane theory. On the other hand, the type IIB string is conjectured to have an $S L(2, \mathbb{Z})$ U-duality symmetry [1], which would imply that it is self-dual: the strong coupling limit is again a type IIB superstring theory [3] in which the RR string of the original theory becomes fundamental, through a ten-dimensional string-string duality [9]. In addition to the NS-NS string which is fundamental at weak coupling, the theory has a NS-NS 5-brane whose density scales as $g^{-2}$ and RR $p$-branes for $p=1,3,5,7,9$ whose densities scale as $g^{-1}$ [23]. String/5brane duality would require that the NS-NS 5-brane governs the strong coupling dynamics, while string-string duality would require that it is the $\mathrm{RR}$ string that does so. However, all the $p$-branes have densities that become small at strong coupling and in particular the NS-NS 5-brane is the one whose density (with respect to the string-metric) tends to zero 'fastest'. It is important to understand the strong coupling dynamics in this case, and in particular to test the predictions of U-duality.

Conjectures for the strong coupling limits of many string theories are to be found in [3], but a number of gaps remain. One of the most interesting concerns the strong coupling behaviour of the type IIB theory compactified on $K_{3}$. As will be seen, going to infinity in certain directions in the coupling constant space of this theory gives limits that do not seem to correspond to any known theories and might arise from a new theory in twelve dimensions.

The purpose of this paper is to address these and related issues. In [3] the strong coupling limit of various string theories was investigated by seeking the 0 brane or particle states that became massless fastest as one went to infinity in a particular direction in coupling constant space. Here, this will be generalised to a study of the $p$-brane states (which may be represented at weak coupling by solitons 
or fundamental branes or D-branes) that become massless or null in superstring or supergravity or $\mathrm{M}$ theories in certain weak or strong coupling limits. It does not make sense to ask which of these $p$-branes become massless 'fastest', since the masses of $p$-branes with different values of $p$ cannot be compared. Instead, the question as to which $p$-branes govern the weak or strong coupling behaviour is addressed by finding a criterion to establish which correspond to perturbative states at weak coupling and which to perturbative states of the strong coupling theory, when considered as a perturbation theory in the inverse coupling. If the perturbative states are one-branes, then the perturbative theory is a string theory, while if they are 0-branes it is a field theory, and so on. The analysis of $[3,5]$ gave the perturbative particle states of the strong coupling limit of various string theories and hence identified the supergravity theory that described the low-energy effective dynamics at strong coupling, the analysis presented here goes some way to identifying the cases in which the perturbation theory that arises in the strong coupling limit is a superstring theory and the cases in which it is a superparticle theory.

The key to the analysis is to study the coupling constant dependence of the mass $/ p$-volume of the $p$-branes. For example, in $N=4$ supersymmetric Yang-Mills in $D=4$ with the gauge group spontaneously broken to an abelian subgroup, the masses are conventionally defined so that particles carrying electric charge have masses that are independent of the coupling constant $g$, while those carrying magnetic charge have $M \sim 1 / g^{2}$, so that in the weak coupling limit $g \rightarrow 0$ the magnetic charges have infinite mass and only the electric charges remain. Perturbation theory is then based on these electric charges (together with neutral particles) and it is these that propagate in Feynman diagrams, while the magnetic charges are treated

* If there are some compact dimensions, one can compare the masses of particle states in the compactified theory arising from $p$-branes wrapping around homology cycles, but it is clear from [3] that the relationship between the strong coupling dynamics of compactified theories and that of uncompactified ones can be subtle, and it is desirable to give an analysis that does not rely on compactification, but which gives the expected results if some dimensions are compactified. 
as solitons. To study strong coupling, it is convenient to make certain rescalings so that the magnetic charges have $M \sim 1$ while the electric charges have $M \sim g^{2}$. Then the electric charges decouple in the strong coupling limit $g \rightarrow \infty$ and are treated as solitons, while the magnetic charges (plus neutral states) are perturbative states for the perturbation theory arising from the expansion in $\hat{g}=1 / g$, which is small for large $g$. There is perhaps an underlying theory in which electric and magnetic charges appear symmetrically, but when treated perturbatively in $g$, the electric charges are perturbative states and the magnetic charges are solitonic, while when treated perturbatively in $\hat{g}$, the magnetic charges are perturbative states and the electric charges are solitonic. The 'perturbative states' are the fundamental objects of the usual field theory description, and the strong coupling field theory is again an $N=4$ super-Yang-Mills theory as expected from the MontonenOlive-Osborne duality conjecture. However, the conventional field theory picture is perhaps an artifice of using perturbation theory and at intermediate values of $g$ field theory is not so useful. Indeed, at enhanced symmetry points both electric and magnetic charges become massless [4], so that a conventional local field theory description cannot be applied. However, until the underlying theory with electric and magnetic charges treated 'democratically' is understood, important progress can be made by studying the effective perturbation theory in $g$ or $\hat{g}$.

As another example, consider the type IIA or IIB string in ten dimensions. In the string metric, the NS-NS string has density $M_{1}$ that is independent of the string coupling $g$, while the RR $p$-branes have density $M_{p} \sim 1 / g$ and the NS-NS 5 -brane has density $M_{5} \sim 1 / g^{2}$. This is consistent with treating the NS-NS string as the perturbative state in an expansion in $g$, so that a conventional string perturbation theory emerges, while the other $p$-branes decouple as $g \rightarrow 0$ and should be treated as solitons.

A similar picture extends to other theories with local supersymmetry, such as superstrings, M-theory or supergravities. The $p$-brane spectrum can be studied by consideration of the low-energy effective supergravity theory. In cases with enough unbroken supersymmetry, the densities of BPS-saturated $p$-branes, and in 
particular their coupling constant dependence, is reliably given by the Bogomolnyi formula. The coupling constant dependence can be changed by rescaling the metric etc, but in each case there are two particularly natural choices of metric etc. In the first, some of the $p$-branes have densities that are independent of a particular coupling constant $g$, and all other densities depend on $g$ to a negative power. This suggests treating the states with $M_{p} \sim 1$ as perturbative states in a weak coupling expansion, and the others with $M_{p} \sim g^{-n}$ (for some $n$ ) as solitons. With the second choice, there is some new set of states with $M_{p} \sim 1$ and all others depend on $g$ to a positive power. This suggests treating the states with $M_{p} \sim 1$ as perturbative states in a strong coupling expansion in $\hat{g}=1 / g$, and the others with $M_{p} \sim \hat{g}^{-n}$ as solitons. In either case, this identifies those BPS-saturated $p$-brane states that should be included in the relevant perturbative spectrum, together with other states including neutral massless ones. These are the states that should propagate in loops. In some cases, the perturbative spectrum consists of strings and a consistent superstring perturbation theory emerges, while in others it is that of a supergravity theory in which case the corresponding perturbation theory only makes sense in the presence of a cut-off. We shall see that this analysis gives the expected results in many cases where alternative methods (e.g. using U-duality) can be used, and gives a consistent picture in other cases.

In the cases examined here, the perturbative theory that emerges is almost always either a supergravity theory or a superstring theory. Moreover, a given theory can look like a superstring theory in the perturbation theory for one coupling constant, or like a supergravity theory when expanded with respect to another. Perturbative $p$-brane theories with $p>1$ do not seem to emerge. However, in each case the analysis depends on knowing the relevant parts of the $p$-brane spectrum, and in some cases our knowledge is incomplete and so the results presented are provisional. In particular, while our knowledge of BPS $p$-brane states is fairly complete, we know very little about non-BPS states, which may also exist in a perturbative spectrum. In some cases, these are metastable in some weak-coupling regime, but cannot be extrapolated to states for other values of the coupling. In 
most cases, a satisfactory picture emerges in terms of BPS states alone, but in some cases it appears that it is the metastable non-BPS states that are perturbative and govern the dynamics in some coupling constant regime.

The results suggest that M-theory or $p$-brane theories such as the 11dimensional supermembrane, if they exist, cannot be treated perturbatively as p-brane theories (indeed, the uncompactified 11-dimensional supermembrane has no coupling constant arising from a scalar expectation value) but that when expanded with respect to a coupling constant such as a compactification modulus, the perturbative theory that emerges is a superstring or supergravity theory. Remarkably, in many supergravity theories, we learn that the perturbative spectrum with respect to certain coupling constants includes strings, and so consistent perturbative quantization requires string theory. Conversely, expanding a superstring theory in a compactification modulus typically leads to a perturbative spectrum which is that of a supergravity theory rather than a superstring theory.

These arguments identify some of the states which should be used in trying to construct a perturbation theory and which should propagate in loops. Whether or not the quantum perturbation theory 'exists' is another matter. For superstrings, a consistent finite perturbation theory is expected to exist, while for supergravity, it exists at best in the presence of a cut-off. At present, we do not have any good definition of what a perturbative $p$-brane theory might be, so it is just as well that no such theory appears to arise in any strong coupling limit. We also do not have any good definition of M-theory, but in the strong coupling limits that are naturally formulated in 11 dimensions, the perturbative states are just the supergravity 0brane multiples and all other states of M-theory appear non-perturbatively. These results would be consistent with the existence of a non-perturbative quantum Mtheory which, when expanded perturbatively with respect to a compactification modulus, gives a perturbative superstring or superparticle theory. Many of the results presented here can be viewed as giving further evidence supporting the conjectures made in [3] for the strong-coupling behaviour of various string theories: the evidence given in [3] was based on studying particle states, and here it is shown 
that when $p$-brane states are also considered, the conclusions remain the same.

\section{Low-Energy Effective Actions}

The low-energy effective action for the heterotic string in $D$ dimensions includes the bosonic terms

$$
\int d^{D} x \sqrt{-g} e^{-2 \Phi}\left(R+4(\partial \Phi)^{2}+\frac{1}{2}(\partial \chi)^{2}-\frac{1}{12} H^{2}-\frac{1}{4} F^{2}\right)
$$

where $\Phi$ is the dilaton, $\chi^{i}$ are the remaining scalar fields with sigma-model action $(\partial \chi)^{2}=g_{i j}(\chi) \partial_{\mu} \chi^{i} \partial^{\mu} \chi^{j}, H$ is the field strength for the two-form potential $b$ and $F$ is the Yang-Mills field strength taking values in the gauge group $G=U(1)^{2(10-D)} \times$ $K$ where $K$ is a group of rank $26-D$. The same action with the $F^{2}$ term omitted describes the NS-NS sector of the type II string in $D$ dimensions. The action (2.1) describes the low-energy heterotic string for small values of the string coupling $g \equiv\left\langle e^{\Phi}\right\rangle$ and we wish to find the dynamics for strong coupling. One approach is to seek a change of variables to an action that might be interpreted as the lowenergy effective action of a dual string or $p$-brane theory with coupling constant

$g^{\prime}=g^{-\beta}$ for some constant $\beta$. If $\beta>0$, this would be consistent with the conjecture that the strong coupling limit of the original theory is the weak coupling limit of the dual theory, and vice versa. As we shall see, this process can lead to more than one candidate for a dual theory, but it is nonetheless useful in locating possible dual theories.

The rescaling

$$
g_{\mu \nu} \rightarrow e^{2 \alpha \Phi} g_{\mu \nu}, \quad \Phi \rightarrow-\beta \Phi
$$

with

$$
\alpha=-\frac{2}{D-6}, \quad \beta=\frac{4}{D-6}
$$


leads to the dual action

$$
\int d^{D} x \sqrt{-g}\left[e^{-2 \Phi}\left(R+\frac{26-D}{4}(\partial \Phi)^{2}+\frac{1}{2}(\partial \chi)^{2}\right)-\frac{1}{12} H^{2}-\frac{1}{4} e^{-\Phi} F^{2}\right]
$$

For $D=10$ and $G=S O(32),(2.4)$ is the low-energy effective action for the type I string, motivating the conjecture that the $S O(32)$ heterotic and type I strings are dual $[3,33]$. For $D>6, \beta>0$ and (2.1) and (2.4) are a possible dual pair of theories, with the weak coupling regime of one corresponding to the strong coupling regime of the other. For $D<6, \beta<0$ and the weak coupling regime of one corresponds to the weak coupling of the other. An equivalence between the corresponding two weakly coupled string theories seems unlikely. For $D=6$, the transformation (2.2), (2.3) is singular. The action (2.4) would be the effective action for a type I string in $D$ dimensions in which all of the group $G$ arose from Chan-Paton factors, or from some generalisation in which the Yang-Mills kinetic term arose at the same order in perturbation theory as the disk diagram. This suggests the conjecture that the heterotic string in $D>6$ dimensions with gauge group $G$ is dual to some generalised type I theory with gauge group $G$. In $D=10$, this would require a new 'generalised type I string' with $E_{8} \times E_{8}$ gauge group, and such a theory has recently been conjectured to arise from an orbifold compactification of 11-dimensional Mtheory [26]. For $D<10$, this new type I theory would appear to be different from the theory obtained by dimensionally reducing the $D=10$ type I string, as this would lead to an action of the form (2.4) with gauge group $G=S O(32)$ (possibly broken by Wilson lines) but with extra abelian gauge fields from the closed string sector: $10-D$ abelian graviphotons with lagrangian $e^{-2 \Phi} F^{2}$ and $10-D$ abelian gauge fields from the $\mathrm{RR}$ two-form with lagrangian $F^{2}$ that do not couple to the dilaton.

The rescaling (2.2) with

$$
\alpha=-\frac{2}{D-4}, \quad \beta=\frac{2}{D-4}
$$


gives the action

$$
\int d^{D} x \sqrt{-g}\left[e^{-2 \Phi}\left(R+\frac{10-D}{4}(\partial \Phi)^{2}+\frac{1}{2}(\partial \chi)^{2}\right)-e^{2 \Phi} \frac{1}{12} H^{2}-\frac{1}{4} F^{2}\right]
$$

and rewriting in terms of the dual $(D-4)$-form potential $\tilde{b}$ with $(D-3)$-form field strength $\tilde{H}=d \tilde{b}$ given by the Hodge dual of the field strength $H$ of the two-form potential

$$
e^{2 \Phi} * H=\tilde{H}
$$

gives

$$
\int d^{D} x \sqrt{-g}\left[e^{-2 \Phi}\left(R+\frac{10-D}{4}(\partial \Phi)^{2}+\frac{1}{2}(\partial \chi)^{2}-\frac{1}{2(D-3) !} \tilde{H}^{2}\right)-\frac{1}{4} F^{2}\right]
$$

As $\tilde{H}$ couples naturally to a $p$-brane with $p=D-5$, it has been suggested that this action be interpreted as the low-energy action for a $p$-brane theory with $p=D-5$ $[30,32]$. This suggests a duality between the heterotic string and a $p$-brane theory for $D>4$ (so that the strong coupling limit of one is the weak coupling limit of the other) $[30,32]$. For $D=6$, this would be a string/string duality, while for $D=10$ this would be a string/five-brane duality. Thus for $D=10$ we have (at least) two candidates for the dual of the heterotic string: a type I theory and a five-brane theory.

Finally, the rescaling (2.2) with

$$
\alpha=-\frac{2}{D-2}, \quad \beta=\frac{4}{D-2}
$$

gives the Einstein frame action

$$
\int d^{D} x \sqrt{-g}\left[R+\frac{2-D}{4}(\partial \Phi)^{2}+\frac{1}{2}(\partial \chi)^{2}-e^{-2 \Phi} \frac{1}{12} H^{2}-\frac{1}{4} e^{-\Phi} F^{2}\right]
$$

Similar results apply for the low-energy effective actions of type II and type I superstrings. In particular, for type II strings the 2-form potential can be dualised 
to a 6-form potential [35], so that a string theory effective action is replaced by a '5-brane effective action', and various other anti-symmetric tensor gauge fields can be dualised [35].

\section{Coupling Constant Dependence in Four Dimensions}

In four dimensions, the toroidally compactified heterotic string at a generic point in its moduli space has gauge group $U(1)^{28}$ so that there are 28 electric charges $q_{I}$ and 28 magnetic charges $p^{I}$ (as defined in [1]). The supergravity field equations are invariant under $K=S L(2 ; \mathbb{R}) \times O(6,22)$ and this is broken down to the integral subgroup $S L(2 ; \mathbb{Z}) \times O(6,22 ; \mathbb{Z})$ of $\mathrm{S}$ and $\mathrm{T}$ dualities by quantum effects $[12,13]$. The scalar fields take values in the coset space

$$
\frac{S L(2 ; \mathbb{R})}{U(1)} \times \frac{O(6,22)}{O(6) \times O(22)}
$$

and the 56 electric and magnetic charges transform as the irreducible $(\mathbf{2}, \mathbf{2 8})$ representation of $S L(2 ; \mathbb{R}) \times O(6,22)$. The $N=4$ supersymmetry algebra has 6 electric and 6 magnetic central charges $\tilde{q}_{I}, \tilde{p}^{I}$ given in terms of the charges $q_{I}, p^{I}$ by $\tilde{q}=K q, \tilde{p}=K p$ where $K$ is a $6 \times 28$ matrix function of the moduli that are given by the asymptotic values of the scalar fields taking values in $O(6,22) / O(6) \times O(22)$. The $N=4$ Bogomolnyi mass formula for BPS saturated states (preserving half the supersymmetry) is $[12,13]$

$$
M^{2}=\left(\begin{array}{ll}
\tilde{p} & \tilde{q}
\end{array}\right) \mathcal{S}\left(\begin{array}{l}
\tilde{p} \\
\tilde{q}
\end{array}\right)
$$

where $M$ is the ADM mass in the Einstein frame and

$$
\mathcal{S}=\frac{1}{\lambda_{2}}\left(\begin{array}{cc}
|\lambda|^{2} & \lambda_{1} \\
\lambda_{1} & 1
\end{array}\right)
$$

is an $S L(2, \mathbb{R})$ matrix depending on $\lambda=\left\langle a+i e^{-2 \Phi}\right\rangle=\lambda_{1}+i \lambda_{2}$, where $a$ is the axion and $\Phi$ is the dilaton. (In the notation of [12,13], $K^{t} K=\frac{1}{16}(M+L)$.) For 
vanishing axion expectation value $\lambda_{1}$, the mass is given by

$$
M^{2}=g^{2} \tilde{q}^{2}+\frac{1}{g^{2}} \tilde{p}^{2}
$$

where $g=\left\langle e^{\Phi}\right\rangle$ is the string coupling constant. For the mass $M_{s}$ measured with respect to the stringy metric $\tilde{g}_{\mu \nu}$, which is given in terms of the Einstein metric $g_{\mu \nu}$ by $\tilde{g}_{\mu \nu}=e^{2 \Phi} g_{\mu \nu}$, the formula (3.3) becomes

$$
M_{s}^{2}=\tilde{q}^{2}+\left(\frac{1}{g^{2}} \tilde{p}\right)^{2}
$$

This form was to be expected, since the electric charges are carried by perturbative string states while the magnetic ones arise from solitons, and the mass of a magnetically charged state has the standard $1 / g^{2}$ coupling constant dependence of a soliton.

The $S L(2, \mathbb{R})$ symmetry acts as

$$
\mathcal{S} \rightarrow \Lambda^{t} \mathcal{S} \Lambda, \quad\left(\begin{array}{c}
\tilde{p} \\
\tilde{q}
\end{array}\right) \rightarrow \Lambda^{-1}\left(\begin{array}{c}
\tilde{p} \\
\tilde{q}
\end{array}\right)
$$

where $\Lambda$ is a $2 \times 2$ matrix in $S L(2, \mathbb{R})$, and $(p, q)$ transforms in the same way as $(\tilde{p}, \tilde{q})$. The Einstein metric $g_{\mu \nu}$ is invariant, and the mass formula (3.1) is manifestly invariant. The $S L(2, \mathbb{Z})$ transformation given by $(3.5)$ with

$$
\Lambda=\left(\begin{array}{cc}
0 & 1 \\
-1 & 0
\end{array}\right)
$$

interchanges electric and magnetic charges while $\lambda \rightarrow-1 / \lambda$. If $\langle a\rangle=0$, then the coupling constant is inverted, $g \rightarrow 1 / g$, and weak and strong coupling regimes are interchanged. Thus the theory is self-dual: the strongly coupled regime can be treated using perturbation theory in the small coupling constant $\hat{g}=1 / g$ and this gives a dual heterotic string theory. In the weakly coupled theory, the electric 
charges $q$ were carried by $g$-perturbative states (i.e. ones that arise in the perturbation theory with respect to $g$ ) and the magnetic ones $p$ by solitons, while in the dual theory the electric charges $q$ are carried by solitons and the magnetic ones $p$ are carried by states that arise as $\hat{g}$-perturbative states.

For the dual theory, it is appropriate to use the dual stringy metric $\tilde{g}_{\mu \nu}$, which is given in terms of the Einstein metric $g_{\mu \nu}$ by $\tilde{g}_{\mu \nu}=e^{-2 \Phi} g_{\mu \nu}$. The mass $M_{d}$ measured with respect to this metric is then, from (3.3),

$$
M_{d}^{2}=\tilde{p}^{2}+\left(\frac{1}{\hat{g}^{2}} \tilde{q}\right)^{2}
$$

This is consistent with the fact that $p$ is carried by $\hat{g}$-perturbative states and $q$ by solitons for $g>>1(\hat{g}<<1)$.

Consider now the type II theory toroidaly compactified to four dimensions. The gauge group is again $U(1)^{28}$, so that there are again $28+28$ electric and magnetic charges that form a 56 -vector $\mathcal{Z}$ which in the quantum theory must take values in a self-dual lattice. The low-energy effective action is that of $N=8$ supergravity [36], which has an $E_{7(7)}$ symmetry of the equations of motion which is conjectured to lead to a discrete $E_{7}(\mathbb{Z})$ U-duality symmetry of the string theory [1]. The charge vector $\mathcal{Z}$ transforms according to the irreducible 56 representation of $E_{7}$, which has the decomposition

$$
56 \rightarrow(2,12)+(1,32)
$$

under the subgroup $S L(2 ; \mathbb{R}) \times S O(6,6)$. This is to be compared with the heterotic string, for which the charge vector $(p, q)$ has the decomposition

$$
(2,28) \rightarrow(2,12)+16 \times(2,1)
$$

in terms of representations of $S L(2 ; \mathbb{R}) \times S O(6,6)$. In both cases there is a common sector corresponding to the $(\mathbf{2}, \mathbf{1 2})$ representation of $S L(2 ; \mathbb{R}) \times S O(6,6)$, plus an additional 32-dimensional representation corresponding, for the heterotic string, to 
the charges for the additional $U(1)^{16}$ gauge group and, for the type II strings, to the charges for the RR sector gauge fields.

The scalar fields take values in the coset space $E_{7} / S U(8)$ and can be represented by a $56 \times 56$ matrix $\mathcal{V}$ that transforms under $E_{7}$ from the right and under local $S U(8)$ transformations from the left [36]. The charge vector $\mathcal{Z}$ enters the Bogomolnyi mass formula through the $E_{7}$-invariant combination $\overline{\mathcal{Z}}=\overline{\mathcal{V}} \mathcal{Z}$, where $\overline{\mathcal{V}}$ is the asymptotic value of $\mathcal{V}$. The Lie algebra of $E_{7}$ can be decomposed into that of $S L(2 ; \mathbb{R}) \times S O(6,6)$ and its orthogonal complement $X$, so that $\overline{\mathcal{V}}$ can be written as $\bar{V}=S T R$ where $S \in S L(2 ; \mathbb{R}), T \in S O(6,6)$ and $R$ is the exponential of an element of $X$. Then the dressed charge vector $\tilde{\mathcal{Z}}=T R \mathcal{Z}$ decomposes into 12 doublets of $S L(2, \mathbb{R})$, consisting of $12+12$ 'dressed' electric and magnetic charges $\left(\tilde{p}^{I}, \tilde{q}_{I}\right)$, together with 32 singlets of $S L(2 ; \mathbb{R})$, the 'dressed' RR charges $\tilde{r}_{a}$. The ADM mass formula for Bogomolnyi states in the Einstein-frame is then

$$
M^{2}=\left(\begin{array}{ll}
\tilde{p} & \tilde{q}
\end{array}\right) \mathcal{S}\left(\begin{array}{l}
\tilde{p} \\
\tilde{q}
\end{array}\right)+\tilde{r}^{2}
$$

where $\mathcal{S}$ is given in terms of $\lambda=\left\langle a+i e^{-2 \Phi}\right\rangle$ by (3.2). The dependence on $\lambda$ can be understood from group theory, and in particular the fact that the RR charges $\tilde{r}$ occur without any dependence on $\lambda$ follows from the fact that they are $S L(2, \mathbb{R})$ singlets. For vanishing $\lambda_{1}$, the mass is given by

$$
M^{2}=g^{2} \tilde{q}^{2}+\frac{1}{g^{2}} \tilde{p}^{2}+\tilde{r}^{2}
$$

For the mass $M_{s}$ measured with respect to the stringy metric $e^{2 \Phi} g_{\mu \nu}$, this becomes

$$
M_{s}^{2}=\tilde{q}^{2}+\left(\frac{1}{g} \tilde{r}\right)^{2}+\left(\frac{1}{g^{2}} \tilde{p}\right)^{2}
$$

while for mass $M_{d}$ corresponding to the dual stringy metric $e^{-2 \Phi} g_{\mu \nu}$ the Bogomolnyi mass formula is

$$
M_{d}^{2}=\tilde{p}^{2}+\left(\frac{1}{\hat{g}} \tilde{r}\right)^{2}+\left(\frac{1}{\hat{g}^{2}} \tilde{q}\right)^{2}
$$

Thus, as in the $N=4$ case, NS-NS electric and magnetic charges are interchanged 
under strong/weak duality, but the states carrying RR charges are non-perturbative at both weak and strong coupling. Whereas magnetic charges are associated with effects with the usual non-perturbative coupling dependence of $e^{-1 / g^{2}}$, the RR charges are associated with ones with the stringy dependence $e^{-1 / g}$, similar to that found in matrix models [37].

\section{Bogomolnyi States and $p$-branes}

The particle states with a given charge that saturate a Bogomolnyi bound are expected to be in the theory for all values of the coupling constants, although they might be represented as elementary excitations for some values of the coupling constants and as solitons for others. For example, in $N=4$ supersymmetric YangMills theory with gauge group spontaneously broken to $U(1)$, the Montonen-Olive duality conjecture implies that the magnetic charges are carried by BPS monopoles at weak coupling, while the same states extrapolated to strong coupling are elementary particles - W-bosons - of the dual $N=4$ Yang-Mills theory describing the strongly coupled theory. There is as yet no clear picture of what happens for intermediate values of the coupling. With enough supersymmetry (at least $N=4$ in four dimensions) the Bogomolnyi states are specified by their charges and their masses are given in terms of these charges and the coupling constants (corresponding to scalar field expectation values) by the Bogomolnyi formula, which is determined completely by considerations of low-energy supersymmetry.

Similarly, there are $p$-brane states in the theory that saturate a Bogomolnyi bound and are also expected to be in the theory for all values of the coupling constants, and again these can be elementary for some values of the couplings and solitonic for others. Here, we shall mainly be concerned with states that break half the supersymmetry. The $p$-brane states of the superstring theory are sources for the long range fields and so lead to field configurations which are the $p$-brane solutions of the low-energy effective supergravity theory. For example, most superstring theories (with the possible exception of type I) have a 1-brane saturating 
a Bogomolnyi bound and which is the fundamental string of the weakly coupled theory but a solitonic string of the strongly coupled dual theory. Most string theories also have Bogomolnyi $p$-branes that, at weak coupling, are either non-singular solitons of the weakly coupled theory or are associated with D-branes, and these should again extrapolate to $p$-brane states at other values of the couplings. The mass per unit $p$-volume is given by the Bogomolnyi formula. This is again determined by low-energy supersymmetry, but it will be convenient here to read off the coupling constant dependence of the $p$-density from that of a $p$-brane solution of the effective field theory and extrapolate the formula to all values of the couplings. This gives the same result as the Bogomolnyi formula, but can also be applied to non-Bogomolnyi solitons, although in the latter case the lack of supersymmetry can allow quantum corrections to the masses and charges.

Consider an action of the form

$$
\int d^{D} x \sqrt{-g}\left(e^{-2 \Phi}\left[R+4(\partial \Phi)^{2}\right]-e^{-\gamma \Phi} \frac{1}{2(n+1) !} G^{2}+\ldots\right)
$$

where $G$ is the $(n+1)$-form field strength for an $n$-form gauge potential $C$ (the case $n=0$ is a scalar field) and $\gamma$ is a constant. Such actions arise as part of superstring effective actions. The constant $\gamma$ takes the values $\gamma=2$ for the heterotic string or for a NS-NS field in a type II string, while $\gamma=0$ for RR fields of type I and II strings and $\gamma=1$ for the type I vector fields (and type I scalars in $D<10$ ) whose kinetic terms arise from a disk diagram. Such theories have $p$-brane solitons which couple to $C$ and carry corresponding charges [28,30]. These consist of electrically charged $(n-1)$-brane solitons and magnetically charged $(D-n-3)$-brane solitons. It is straightforward to check (cf [30]) that the mass per unit $p$-volume $M_{p}$ of a $p$-brane soliton of this type has the following coupling constant dependence:

$$
M_{p} \sim g^{-\epsilon_{p}} T_{p}, \quad \epsilon_{p}= \begin{cases}1-\frac{\gamma}{2} & \text { for electric } p \text {-branes } \\ 1+\frac{\gamma}{2} & \text { for magnetic } p \text {-branes }\end{cases}
$$

Here the coupling constant $g$ is given by the asymptotic value of $e^{\Phi}$ (which is assumed constant) and $T_{p}$ is a dimensionful constant that can be thought of as 
an effective brane tension, and which contains the dependence on any coupling constants other than $g$. In the case $n=0, C$ is a scalar field which formally appears to couple to an electric $p$-brane with $p=-1$. This can be interpreted as an instanton in the Euclidean version of the theory $[23,38]$, but this case will not be considered further here as we will be interested in the $p$-brane spectrum for Lorentzian signature. The scalar also couples to a magnetic $(D-3)$-brane (e.g. a string in $D=4$ or a 7 -brane in $D=10[38]$ ) and these will be considered here.

Configurations satisfying $p$-brane boundary conditions typically satisfy a Bogomolnyi bound of the form $M_{p} \geq|\mathcal{Z}|$ where $M_{p}$ is the mass per unit $p$-volume and $|\mathcal{Z}|$ is the electric or magnetic central charge, which is given in terms of the asymptotic values of the scalar fields and the magnetic charge $P=\int_{S^{n+1}} G$ (given by an integral over an $(n+1)$-sphere surrounding the $(n-1)$-brane) or the electric charge $Q=\int_{S^{D-n-1}} e^{-\gamma \Phi} * G$ (integrated over a $(D-n-1)$-sphere surrounding the $(D-n-3)$-brane). The precise coupling constant dependence of the relation between $\mathcal{Z}$ and $Q, P$ is determined by supersymmetry, and the dependence on scalars other than $\Phi$ has been suppressed. Of particular interest are those $p$-brane solitons that break half the supersymmetry, and consequently saturate the Bogomolnyi bound so that the density is given by $M_{p}=|\mathcal{Z}|[27,30]$. This takes the form (4.2), but in this case the relation is expected to be exact so that the density of the corresponding quantum state is given by (4.2).

The mass formula (4.2) is valid for the string metric $g_{\mu \nu}$, but it is straightforward to generalise to an arbitrary metric $\tilde{g}_{\mu \nu}$ given by

$$
\tilde{g}_{\mu \nu}=e^{2 \alpha \Phi} g_{\mu \nu}
$$

Lengths and masses as measured in the asymptotic region (where $e^{\Phi} \sim g$ ) with respect to the two metrics are related by $\tilde{L}=g^{\alpha} L, \tilde{M}=g^{-\alpha} M$ so that the masses per unit $p$-volume are related by

$$
\tilde{M}_{p}=g^{-(p+1) \alpha} M_{p}
$$


Then for a general metric $\tilde{g}_{\mu \nu}(4.2)$ becomes

$$
M_{p} \sim g^{-\left[\epsilon_{p}+(p+1) \alpha\right]} T_{p}, \quad \epsilon_{p}= \begin{cases}1-\frac{\gamma}{2} & \text { for electric } p \text {-branes } \\ 1+\frac{\gamma}{2} & \text { for magnetic } p \text {-branes }\end{cases}
$$

For $\alpha=-2 /(D-2), \tilde{g}_{\mu \nu}$ is the Einstein metric and (4.5) reproduces the results of [30].

The $g$-dependence of the masses clearly depends on the choice of metric. In the string metric $\alpha=0$, the ratio $M_{p} / T_{p}$ is independent of $g$ for the fundamental string of some string theory $\left(\epsilon_{p}=0\right.$ and $\left.\gamma=2\right)$, while for the corresponding magnetic $(D-5)$-brane soliton the ratio $M_{p} / T_{p}$ is proportional to $g^{-2}$ and for $\mathrm{RR}$ branes it is proportional to $g^{-1}$. Thus in the limit $g \rightarrow 0$, the solitons and D-branes become infinitely massive and decouple, leaving the fundamental string in the perturbative spectrum. This is precisely what is required for a sensible perturbation theory in some small coupling constant $\lambda$ (which might be the string coupling or be associated with the expectation of some other scalar): some states with masses that are independent of $\lambda$ and which are the perturbative states of the theory, with all other states having masses that tend to infinity as $\lambda \rightarrow 0$ so that they are non-perturbative states. This can now be applied to the strong coupling limit. As we shall see, for any supergravity or superstring theory there is a unique choice of rescaled metric (4.3) such that the ratio $M_{p} / T_{p}$ in that metric is independent of $g$ for some states and tends to zero as $g \rightarrow \infty$ for all other states, so that they decouple in the strong coupling limit. There is then a sensible perturbation theory in $\hat{g}=1 / g$ with the perturbative states consisting of those with $g$-independent masses in the 'dual metric'. Note that the set of $\hat{g}$-perturbative states identified in this way is not the same as the set of non-perturbative states of the original string theory, i.e. those states for which the string-metric ratio $M_{p} / T_{p}$ tends to infinity as $\hat{g} \rightarrow 0$. More generally, as we shall see, given any supergravity or superstring theory and any choice of coupling constant $\lambda$, there are precisely two preferred rescaled metrics, one which leads to a sensible non-trivial weak-coupling perturbation theory in $\lambda$ of the type described above, and one which leads to 
a sensible non-trivial strong-coupling perturbation theory in $1 / \lambda$. Of course the theory can be studied in whichever metric one chooses. The point here is that the preferred metrics are the ones in which the perturbation theory looks most natural, with the perturbative states having $\lambda$-independent masses. It is conceivable that other choices of metric might lead to consistent perturbation theories in this way, but these would be non-maximal, as the perturbative spectrum would be strictly smaller than that arising from one of the two preferred metrics, as will be seen in the examples below.

In this way, it is possible to identify the part of the perturbative spectrum consisting of BPS-saturated $p$-branes that emerges at strong coupling and at weak coupling in lambda for any given theory. We shall first check that this gives the expected results in cases where U-duality gives an alternative derivation of strongcoupling dynamics. It will then be applied to other cases, and in particular to those theories described in the introduction, where it will help decide between different conjectures for the strong-coupling dynamics.

\subsection{Type II in Four Dimensions}

Consider the example of the four-dimensional type II string (compactified on a torus) for which the masses of Bogomolnyi 0-branes is given by (3.12) in the stringy metric. The $g$-perturbative states are those carrying NS-NS electric charge $\tilde{q}$ and have $g$-independent masses $M_{s}=\tilde{q}$, while the non-perturbative states carrying NSNS magnetic or RR charges have masses $M_{s}=g^{-2} \tilde{p}$ and $M_{s}=g^{-1} \tilde{r}$ respectively and become massless at strong coupling, $M_{s} \rightarrow 0$ as $g \rightarrow \infty$. However, the NS-NS magnetic states become massless fastest and it is these that become the $\hat{g}$-perturbative states at strong coupling $(\hat{g}=1 / g)$. For a general metric (4.3), (3.12) becomes

$$
M^{2}=\left[g^{-\alpha} \tilde{q}\right]^{2}+\left[g^{-(1+\alpha)} \tilde{r}\right]^{2}+\left[g^{-(2+\alpha)} \tilde{p}\right]^{2}
$$

so that for states carrying only one type of charge, the masses are $M=g^{-\alpha} \tilde{q}$, $M=g^{-(1+\alpha)} \tilde{r}$ or $M=g^{-(2+\alpha)} \tilde{p}$. 
There are two preferred values of $\alpha$ which give good perturbation theories. The stringy metric $\alpha=0$ gives the perturbative spectrum described above. The value $\alpha=-2$ gives the 'dual stringy metric' for which NS-NS magnetically charged states have $M=\tilde{p}$ and are perturbative, while electric states have $M=\hat{g}^{-2} \tilde{q}$ and RR ones have $M=\hat{g}^{-1} \tilde{r}$. States carrying $\tilde{q}$ or $\tilde{r}$ charge (including those with more than one type of charge) have masses that diverge in the strong-coupling limit $\hat{g}=1 / g \rightarrow 0$ and so are non-perturbative in the strong-coupling perturbation theory. For metrics with $-2<\alpha<0$ there are states that become massless as either $g \rightarrow 0$ or $g \rightarrow \infty$ so that there is no consistent weak or strong coupling perturbation theory. In particular, for the Einstein metric $\alpha=-1$ the RR states have $g$-independent masses but there is no consistent weak or strong coupling perturbation theory based on the RR states and neutral states alone. For $\alpha>0$, the Bogomolnyi formula (4.6) implies that all charged states have masses that depend on a negative power of $g$, so that they decouple in the $g \rightarrow 0$ limit and any states with $g$-independent masses must be uncharged. Thus a weak-coupling perturbation theory, if consistent, would be non-maximal as it would contain no perturbative charged states, and would correspond to the limit of the usual perturbation theory in which the unit of charge is taken to infinity. In the strong coupling limit with $\alpha>0$, all charged states become massless so that it would not make sense to base a perturbation theory on uncharged states alone. Similar considerations apply for $\alpha<-2$, for which there is a strong coupling perturbation theory involving no charged states. This leads us to the two preferred metrics with $\alpha=0,-2$ and the corresponding weak and strong coupling perturbation theories. This is precisely the result predicted by U-duality: the theory is self-dual, with the strong coupling theory given by the a dual version of the original theory with NS-NS electric and magnetic charges interchanged. 


\subsection{Type IIB IN $D=10$}

Type IIB supergravity and the type IIB superstring in $D=10$ both have two 1-brane solutions, two 5-brane solutions and a self-dual 3-brane solution [28], together with a 7 -brane and a 9-brane $[23,38]$. (We will only be interested in the Lorentzian signature solutions here and so will not consider the instantonic -1 brane [38].) The values of $p, \gamma, \epsilon_{p}$ and $\rho_{p} \equiv \epsilon_{p} / p+1$ are given in the following table:

\begin{tabular}{|c|c|c|c|c|c|}
\hline Value of $p$ & 1 (NS-NS) & 5 (NS-NS) & $1^{\prime}(\mathrm{RR})$ & $5^{\prime}(\mathrm{RR})$ & $3^{+}(\mathrm{RR})$ \\
\hline$\gamma$ & 2 & 2 & 0 & 0 & 0 \\
\hline$\epsilon_{p}$ & 0 & 2 & 1 & 1 & 1 \\
\hline$\rho_{p}=\epsilon_{p} / p+1$ & 0 & $1 / 3$ & $1 / 2$ & $1 / 6$ & $1 / 4$ \\
\hline String metric $M_{p} / T_{p}$ & 1 & $g^{-2}$ & $g^{-1}$ & $g^{-1}$ & $g^{-1}$ \\
\hline Dual string metric $M_{p} / T_{p}$ & $g$ & $g$ & 1 & $g^{2}$ & $g$ \\
\hline Einstein metric $M_{p} / T_{p}$ & $g^{1 / 2}$ & $g^{-1 / 2}$ & $g^{-1 / 2}$ & $g^{1 / 2}$ & 1 \\
\hline General metric $M_{p} / T_{p}$ & $g^{-2 \alpha}$ & $g^{-6(1 / 3+\alpha)}$ & $g^{-2(1 / 2+\alpha)}$ & $g^{-6(1 / 6+\alpha)}$ & $g^{-4(1 / 4+\alpha)}$ \\
\hline
\end{tabular}

Table 1 Type IIB Brane-Scan.

The 1-branes are electric, the 5-branes are magnetic and the self-dual 3-brane has equal electric and magnetic charges. Those with $\gamma=2$ couple to the NS-NS 2 -form potential while those with $\gamma=0$ couple to RR gauge fields. (There is no action of the form (4.1) for the 4-form potential with self-dual field strength, but the mass formula (4.2) can nonetheless be applied to the 3-brane with $\gamma=0$ [30].) This can be extended to include the 7 and 9 branes. It follows from [23] that the RR $p$-branes with $p=1,3,5,7,9$ all have $M_{p} / T_{p}$ given by $g^{-1}$ in the string metric and so by $g^{-[1+(p+1) \alpha]}$ with respect to the general metric, corresponding to $\rho_{p}=1 / p+1$.

In each case, the $p$-brane mass satisfies

$$
M_{p} \sim g^{-(p+1)\left[\rho_{p}+\alpha\right]} T_{p}
$$

and for a given $p$-brane, this is $g$-independent for the metric (4.3) with $\alpha=-\rho_{p}$. 
For each such $p$-brane metric except those the preferred cases $\alpha=0,-1 / 2$, some of the soliton masses grow with $g$ and others grow with $1 / g$, so that there is no sensible weak or strong coupling perturbation theory; this is seen explicitly in the table for the Einstein metric $(\alpha=-1 / 4)$ for which the self-dual 3-brane has $g$ independent mass. The special cases $\alpha=0,-1 / 2$ are the minimum and maximum values of $\rho_{p}$ and give masses that depend on $g$ raised to a non-positive power or to a non-negative one, respectively. For $\alpha=0$, the $g$-perturbative Bogomolnyi spectrum consists of the 1-brane with $\gamma=2$, which is the fundamental string so that we learn that the perturbation theory is that of a string theory. Note that even if we had started with the IIB supergravity theory (including $p$-brane solitons), we would have learned that the weakly coupled theory is a perturbative superstring theory, in which the $\gamma=2$ soliton is identified with the fundamental string. All other $p$-brane densities depend on $g$ to a negative power and so correspond to non-perturbative states whose (string metric) densities tend to infinity at weak coupling.

For strong coupling, we learn that the appropriate metric for describing the dual theory is (4.3) with $\alpha=-1 / 2$, and that the $1^{\prime}$-brane which couples to the $\gamma=$ 02 -form becomes $\hat{g}$-perturbative and is the fundamental string for the dual theory, while the other $p$-branes become $\hat{g}$-non-perturbative states of the dual theory. Thus the dual theory is again a string theory. This is in complete agreement with the conjecture that the type IIB string has an $S L(2 ; \mathbb{Z})$ duality symmetry [1] and so is self-dual: the strong coupling limit is a dual type IIB string theory in which NS-NS and RR charges have been interchanged. Thus although in the original string-metric, the NS-NS 5-brane has a mass which tends to zero as $g^{-2}$ at strong coupling and the RR branes have masses that tend to zero as $g^{-1}$, it is the RR string that here wins out over the others and dominates the strong coupling theory, so that the theory is self-dual rather than dual to e.g. a 5-brane theory.

Mass formulae similar to those given in the last section for the $D=4$ heterotic string, which is also self-dual with an $S L(2 ; \mathbb{Z})$ symmetry, can be written down for the type IIB theory. A 1-brane can carry an NS-NS charge $q$ (coupling to the 
NS-NS 2-form) or a RR charge $r$ (coupling to the RR 2-form) or both, in which case it is 'dyonic'. (Dyonic solutions can be constructed from the NS-NS string and RR string given in [9] by acting with $S L(2 ; \mathbb{Z})$.) The Einstein frame mass per unit length $M_{1}$ satisfies a Bogomolnyi bound which, for vanishing pseudo-scalar, is

$$
M_{1}^{2} \geq g q^{2}+g^{-1} r^{2}
$$

Strings saturating this bound have masses saturating this bound have masses satisfying

$$
M_{1}^{2}=q^{2}+g^{-2} r^{2}
$$

in the string frame or

$$
M_{1}^{2}=r^{2}+\hat{g}^{-2} q^{2}
$$

in the dual string frame.

\subsection{The General Case}

Consider now an arbitrary supersymmetric theory in $D$ dimensions, which might be a supergravity, superstring or super-p-brane theory. Usually, we will restrict ourselves to situations in which there is enough supersymmetry for the classical Bogomolnyi formulae to be reliable in the quantum theory. Let $\phi$ be some scalar field, which might be the dilaton $\Phi$, or $-\Phi$, or some compactification modulus. Then $g=e^{\langle\phi\rangle}$ is one of the coupling constants of the theory and one can attempt to define perturbation theory with respect to $g$. There is a spectrum of $p$-branes, and for each $p$-brane there is a constant $\tilde{\rho}_{p}$ such that the mass per unit $p$-volume has a dependence on the coupling constant $g$ that is given with respect to the Einstein metric by

$$
M_{p} \sim g^{-(p+1) \tilde{\rho}_{p}} T_{p}
$$

The dependence on all other coupling constants is absorbed into the effective tension $T_{p}$. In string theory, the usual weak coupling perturbation expansion is that 
in $\left\langle e^{\Phi}\right\rangle$, but expanding in other couplings such as $\left\langle e^{-\Phi}\right\rangle$ or one of the compactification moduli can give new insights and can lead to a new perturbative theory, which might be another string theory or a supergravity theory or perhaps something more exotic such as a p-brane theory. (The 11-dimensional supergravity or supermembrane theory has no scalars or coupling constants and so this analysis does not apply. If the 11-dimensional theory is compactified, however, one of the compactification moduli can be used as a coupling constant and a mass formula (4.11) can be found.)

If the Einstein metric is conformally rescaled to

$$
g_{\mu \nu}^{\prime}=e^{2 \tilde{\alpha} \phi} g_{\mu \nu}
$$

then since the conformal factor tends to $g^{2 \tilde{\alpha}}$ in the asymptotic region, the mass formula becomes

$$
M_{p} \sim g^{-(p+1)\left[\tilde{\rho}_{p}+\tilde{\alpha}\right]} T_{p}
$$

with respect to the rescaled metric. The weak coupling regime $g<<1$ is dominated by the brane or branes for which $\tilde{\rho}_{p}$ takes its minimum value $\tilde{\rho}_{\text {min }}$ and the appropriate metric is that given by (4.12) with $\tilde{\alpha}=-\tilde{\rho}_{m i n}$. The branes with $\tilde{\rho}_{p}=\tilde{\rho}_{\text {min }}$ are then the $g$-perturbative states, while the others are $g$-non-perturbative. For example, if the only brane with $\tilde{\rho}_{p}=\tilde{\rho}_{\text {min }}$ is a 1-brane, then the weakly coupled theory is a string theory, while if the only such states are 0-branes, then the perturbative theory is a supergravity theory. Similarly, the strong coupling regime $\hat{g}<<1$ with $\hat{g}=1 / g$ is dominated by the brane or branes for which $\tilde{\rho}_{p}$ takes its maximum value $\tilde{\rho}_{\max }$ and the appropriate metric is that given by $\tilde{\alpha}=-\tilde{\rho}_{\max }$, and again this might be a string theory or a $p$-brane theory or a field theory or a theory of a set of coupled $p$-branes with various values of $p$.

It will be useful to define

$$
\rho_{p}=\tilde{\rho}_{p}+\frac{2}{D-2}, \quad \alpha=\tilde{\alpha}-\frac{2}{D-2}
$$


so that (4.11) becomes

$$
M_{p} \sim g^{-(p+1)\left[\rho_{p}+\alpha\right]} T_{p}
$$

Whereas $\tilde{\alpha}=0$ corresponds to the Einstein metric, $\alpha=0$ corresponds to the 'string metric' for the 'dilaton' $\phi$. Clearly the maximum and minimum values of $\rho_{p}$ correspond to the maximum and minimum values of $\tilde{\rho}_{p}$.

In the remainder of this paper, this structure will be explored in various theories. In each case, there are two preferred metrics corresponding to $\tilde{\alpha}=-\tilde{\rho}_{\text {min }}$ and $\tilde{\alpha}=-\tilde{\rho}_{\max }$ (or equivalently $\alpha=-\rho_{\min }$ and $\alpha=-\rho_{\max }$ ) for weak and strong coupling respectively and the corresponding perturbative spectrum can be read off in each case. This will enable us to decide between various alternative duality conjectures, such as those discussed in the introduction. Before proceeding, however, a number of remarks are in order.

Firstly, the $p$-branes fit into supermultiplets and the formula (4.15) applies to the whole supermultiplet, although we shall usually refer explicitly to only the member of the supermultiplet with lowest spin. Secondly, given a $p$-brane with $M_{p} \sim g^{a} T_{p}$ for some $a$, there will be further states with $M_{p} \sim n g^{a} T_{p}$ for integers $n$ and these are degenerate in mass with a configuration of $n p$-branes which each have $M_{p} \sim g^{a} T_{p}$. Often in what follows, we will take $T_{p}$ to be the minimum value, corresponding to elementary solitons, and not consider the 'bound states' with $M_{p}=n T_{p}$ explicitly. Thirdly, the argument given above will identify only the charged massive BPS states in the perturbative spectrum. There will also be neutral states in the perturbative spectrum, and in particular these can include particles with zero mass and $p$-branes with zero density. In some cases, there are non-BPS states that are metastable perturbative states in some coupling constant regime, even though they cannot be continued to states at other values of the coupling. When these are included in the $p$-brane spectrum, they can 'win' over other $p$-branes and so need to be taken into account. However, in most cases, they lose out to BPS states and in those cases will not be considered explicitly. 


\section{Ten and Eleven Dimensional Examples}

\subsection{Type IIA IN $D=10$}

As discussed in the introduction, there are at least two rival proposals for the strong coupling limit of the type IIA string, a 5-brane theory or an 11-dimensional theory, and the analysis here will enable us to decide between them. Type IIA supergravity and the type IIA superstring both have a 1-brane and a 5-brane (coupling to the 2-form in the NS-NS sector of the string) plus $p$-branes with $p=0,3,4,6,8$ (coupling to $\mathrm{RR}$ fields of the string). The relevant properties are summarised in the following table.

\begin{tabular}{|c|c|c|c|c|c|c|}
\hline Value of $p$ & 1 & 5 & 0 & 2 & 4 & 6 \\
\hline$\gamma$ & 2 & 2 & 0 & 0 & 0 & 0 \\
\hline$\epsilon_{p}$ & 0 & 2 & 1 & 1 & 1 & 1 \\
\hline$\rho_{p}=\epsilon_{p} / p+1$ & 0 & $1 / 3$ & 1 & $1 / 4$ & $1 / 5$ & $1 / 7$ \\
\hline$M_{p} / T_{p}$ in string metric & 1 & $g^{-2}$ & $g^{-1}$ & $g^{-1}$ & $g^{-1}$ & $g^{-1}$ \\
\hline$M_{p} / T_{p}$ in dual string metric & $g$ & $g$ & 1 & $g^{3}$ & $g^{4}$ & $g^{6}$ \\
\hline$M_{p} / T_{p}$ in Einstein metric & $g$ & $g$ & $g^{-1 / 2}$ & $g$ & $g^{3 / 2}$ & $g^{5 / 2}$ \\
\hline$M_{p} / T_{p}$ in general metric & $g^{-2 \alpha}$ & $g^{-(2+6 \alpha)}$ & $g^{-(1+\alpha)}$ & $g^{-(1+4 \alpha)}$ & $g^{-(1+5 \alpha)}$ & $g^{-(1+7 \alpha)}$ \\
\hline
\end{tabular}

Table 2 Type IIA Brane-Scan.

In addition, there is an 8-brane solution of the massive type IIA theory [34], which formally has $M_{p} / T_{p}=g^{-1}$ in the string metric [23], corresponding to $\rho_{8}=1 / 9$. The minimum value of $\rho$ is $\rho_{\min }=0$, corresponding to the 1 -brane which becomes the fundamental string of the weakly coupled perturbative theory, for which the appropriate metric is the stringy metric with $\alpha=0$. This is just as expected. The other $p$-branes all have densities that tend to zero as $g \rightarrow \infty$, so that arguments similar to those of [3] might suggest that all could be important for the strong coupling dynamics. However, as $\rho_{\max }=1$, the 0 -brane multiplet dominates at strong coupling, which is then a $\hat{g}$-perturbative supersymmetric field theory. The BPS-saturated $p$-branes occuring in the strong coupling perturbative spectrum 
are then the the 0-branes, which fit into short massive IIA supermultiplets with spins ranging from 0 to 2 . There is such a multiplet with electric charge $n q_{0}$ and mass $M=\left|n q_{0}\right| / g^{1+\alpha}$ for each integer $n \neq 0$, where $q_{0}$ is some unit of charge [3]. In addition, there is the neutral massless type IIA supergravity multiplet

(corresponding to $n=0$ ) and these constitute the fundamental excitations of the dual theory. Thus the states that emerge in strong coupling perturbation theory are those of the $D=10$ type IIA supergravity theory, plus an infinite tower of short massive multiplets. As we shall see in the next section, this is the perturbative spectrum that emerges from 11-dimensional supergravity compactified on a circle, when the radius of the compact dimension is treated as a coupling constant.

\subsection{1-Dimensional Supergravity, M-Theory and Supermembranes}

11-dimensional supergravity has a 2-brane [39] and a 5-brane soliton [40], but there are no scalar fields whose expectation values can be used as coupling constants. Thus there is no perturbation theory in 11 dimensions that can be used to apply the analysis developed above. However, if the theory is compactified, then the moduli of the compactification space can be used as coupling constants. For example consider compactification on a circle. The radius $R$ can be used as a coupling constant: the massless spectrum consists of a $\mathrm{D}=10$ type IIA supergravity multiplet, and there are in addition Kaluza-Klein momentum modes with mass $M \sim q_{0} n / R$ for integers $n$ and some constant $q_{0}$ (with respect to the Kaluza-Klein metric [3]). These fit into massive supermultiplets that saturate a Bogomolnyi bound and have spins ranging from 0 to 2 , and have masses that tend to zero in the large $R$ limit. These have magnetic partners, which arise as generalised KaluzaKlein monopoles in 11 dimensions and lead to 6-brane solutions on reducing to the 10-dimensional theory [2]. In addition, the 11-dimensional 2 and 5 branes give rise to 10 -dimensional $1,2,4$ and 5 branes. This gives rise to precisely the type IIA brane scan given in table 2 after a general rescaling of the metric, with $g=R^{3 / 2}$, with the Kaluza-Klein metric corresponding to $\alpha=-1 / 3$. Thus for large $R$, the coupling constant $g=R^{3 / 2}$ is large and the 0 -brane multiplet dominates. Then 
the perturbative states consist of a massless type IIA supergravity multiplet, plus an infinite Kaluza-Klein tower of charged ones.

Consider now the behaviour for small $R$. For the compactified 11-dimensional supergravity alone without $p$-branes, the Kaluza-Klein states with $M \sim q_{0} n / R$ are non-perturbative in the expansion in $R$, and the resulting perturbative theory is of course the type IIA supergravity theory in 10 dimensions. However, if the $p$ branes are included in the spectrum, we see that at small $R$ the 1-brane dominates and the perturbation theory is that of the type IIA string theory, so that the 11dimensional theory compactified on a small circle should be treated as a string theory, not a field theory. If there is an 11-dimensional supermembrane or $\mathrm{M}$ theory whose low energy effective action is 11-dimensional supergravity, it should also have a 2-brane and a 5-brane soliton. On compactifying to 10 dimensions, the brane-scan would then again be that of table 2 , so that the small $R$ limit would give the perturbative states of type IIA string theory, while the large $R$ limit would give type IIA supergravity.

If instead the theory is compactified on an $n$-torus of volume $V$, the perturbative BPS states that emerge in perturbation theory in $1 / V$ consist of $n 0$-brane multiplets in $11-n$ dimensions, which transform as an $\mathbf{n}$ under the action of $S L(n)$. Each of these multiplets is the base of a Kaluza-Klein tower. The mapping class group of the torus is the discrete subgroup $S L(n ; \mathbb{Z})$ and this is part of the U-duality group of the compactified theory.

\subsection{The Type I String and Heterotic Strings in $D=10$}

Consider the ten-dimensional $N=1$ supergravity theory coupled to $S O(32)$ super-Yang-Mills, which is the low-energy effective field theory of both the type I string and the $\operatorname{Spin}(32) / \mathbb{Z}_{2}$ heterotic string theories. The heterotic string form of the action (2.1) is related to the type I form (2.4) by the field redefinition (2.2),(2.3). This includes a dilaton sign flip, so that the strong coupling limit of one effective field theory is the weak coupling limit of the other [3]. The theory 
has an electric 1-brane and magnetic 5-brane solution which couple to $b_{\mu \nu}$ and saturate a Bogomolnyi bound. There are also electric 0-branes and magnetic 6branes coupling to Yang-Mills fields taking values in a Cartan sub-algebra of $G=$ $S O(32)$ [28], but these do not preserve any supersymmetries so that their status is unclear. The 1-brane is the fundamental heterotic string and has a mass per unit length which is independent of $g=e^{\langle\Phi\rangle}$ in the natural heterotic string metric. The 0-branes also have $g$-independent classical masses with respect to the heterotic string metric, although these may acquire quantum corrections. The perturbative spectrum of the heterotic string then consists of the string and possibly the 0branes, corresponding to the minimum value of $\rho$. The maximum value of $\rho$ for these $p$-branes occurs for the 5 -brane, so that if there are no $p$-branes in the theory other than those listed above, this would suggest that the strong coupling limit of the heterotic string is a perturbative 5-brane theory. However, there is reason to believe that there may be other $p$-branes in the theory that have higher values of $\rho_{p}$, and if this were the case these would be the strong coupling perturbative states instead of the 5-branes.

The perturbative type I string has open and closed fundamental string states. These strings can break and so are not stable and should not be expected to saturate any BPS bound. However, these give metastable states that should be included in the spectrum at weak coupling. A macroscopic type I string would break, although an unstable solution of the supergravity theory representing the field configuration outside a macroscopic type I string could still exist, at least in the weak coupling limit. However, as the type I string is not BPS saturated, there is no reason to expect that such states could be extrapolated to strong coupling. Indeed, type I strings become more likely to break as the coupling increases, so that such states should not exist at strong coupling, and there should be no corresponding states in the weakly coupled heterotic string. Presumably, such a fundamental type I string should have mass/length that is independent of $g$ in the type I string metric. The mass/length of this type I string would then be $M_{1} \sim g^{-1} T_{1}$ in the heterotic string metric and $M_{1} \sim g^{-1-2 \alpha} T_{1}$ in a general metric given by scaling 
the heterotic metric by $e^{2 \alpha \Phi}$. The brane-scan for all these $p$-branes is

\begin{tabular}{|c|c|c|c|c|c|}
\hline Value of $p$ & 1 (type I) & 1 & 5 & 0 & 6 \\
\hline$\epsilon_{p}$ & 1 & 0 & 2 & 0 & 2 \\
\hline$\rho_{p}=\epsilon_{p} / p+1$ & $1 / 2$ & 0 & $1 / 3$ & 0 & $2 / 7$ \\
\hline$M_{p} / T_{p}$ in heterotic string metric & $g^{-1}$ & 1 & $g^{-2}$ & 1 & $g^{-2}$ \\
\hline$M_{p} / T_{p}$ in type I string metric & 1 & $g^{1}$ & $g^{1}$ & $g^{1 / 2}$ & $g^{3 / 2}$ \\
\hline$M_{p} / T_{p}$ in 5-brane metric & $g^{-1 / 3}$ & $g^{2 / 3}$ & 1 & $g^{1 / 3}$ & $g^{1 / 3}$ \\
\hline$M_{p} / T_{p}$ in general metric & $g^{-(1+2 \alpha)}$ & $g^{-2 \alpha}$ & $g^{-(2+6 \alpha)}$ & $g^{-\alpha}$ & $g^{-(2+7 \alpha)}$ \\
\hline
\end{tabular}

Table 3 Heterotic/Type I Brane-Scan.

This table also includes the coupling constant dependence in the so-called 5-brane metric given in terms of the heterotic string metric by (4.3) with $\alpha=-1 / 3$. Note the unusual $g$-dependence of the 0 and 6 branes in the type I metric.

The maximum and minimum values of $\rho$ are indeed those corresponding to the type I and heterotic strings and the perturbative heterotic and type I strings are a dual pair with the strong coupling regime of one corresponding to the weak coupling regime of the other. There may be other $p$-branes of the theory, but it seems unlikely that they will have $\rho<0$ or $\rho>1 / 2$. Indeed, if there were $p$ branes with such values of $\rho$, then it seems likely that there would be problems for the perturbative formulation of either the heterotic or type I string, and no such difficulties are apparent.

If the metastable type I string were not included in the brane-scan in this way for weak type-I coupling, then it would be hard to find a plausible duality picture unless there were some other as yet unsuspected $p$-brane states of the theory. For example, if there was no type I string states in the heterotic string at strong coupling and no other states with $\rho<1 / 6$, then table 3 would support the conjecture that the strong coupling limit of the heterotic string is a perturbative 5 -brane theory, and presumably the weakly coupled string would correspond to the strongly coupled 5-brane [30,32]. However, table 3 would still support the 
conjecture that the strong coupling limit of the type I string is a weakly coupled heterotic string, and this would then imply the implausible statement that the weakly coupled type I string is equivalent to weakly coupled perturbative 5-brane theory. One way out of this would be if the heterotic string solution of the type I theory $[9,10]$ were not to be regarded as an acceptable soliton solution (perhaps because of its singularity structure [25]) but it has recently been shown that the heterotic string indeed arises as a D-brane in the type I string theory, making the conjecture of the existence of a type I soliton of the heterotic string all the more attractive.

\section{Compactified Type II String Theories}

Toroidally compactified type II superstring or supergravity theories have a number of massless scalar fields and their expectation values can all be regarded as coupling constants. Any of these can be used to define a perturbation theory and one can ask which of the $p$-branes are perturbative states for a given coupling constant. In this section we will consider this for the scalar field corresponding to the dilaton in ten dimensions, while the other coupling constants will be discussed in the next section. In each case, we will examine the $p$-brane spectrum of the appropriate supergravity theory and look for the perturbative states. We will find that for the 'stringy' coupling constant corresponding to the dilaton in ten dimensions, the perturbative spectrum at weak coupling is that of a string theory, while in almost all other cases (for strong stringy coupling or for other coupling constants) the perturbative states are those of a field theory. The main exception is in six dimensions, where the perturbative spectrum at strong string coupling is again that of a string theory. This then supports string/string duality in $D=6$ dimensions, but not string/( $D-5)$-brane duality in $D>6$ dimensions. More precisely, the stringy strong coupling limit in $D>6$ is some theory whose perturbative states are particles, not strings or $p$-branes. It will have $p$-brane solitons, but is not a perturbative $p$-brane theory involving the sum over $p$-brane world-volume topologies etc. 
In $D$ dimensions, a $(p+1)$-form gauge field couples to an electric $p$-brane and a magnetic $\tilde{p}$-brane, where $\tilde{p}=D-p-4$. On toroidally compactifying to $D$ dimensions, the NS/NS 2-form gauge field $b_{\mu \nu}$ gives rise to a D-dimensional 2-form, $d=10-D$ vector gauge fields and $d(d-1) / 2$ scalars, while the metric gives rise to an additional $d$ vectors and $d(d+1) / 2$ scalars. The 2 -form couples to a string and a $(D-5)$-brane and the $2 d$ vectors couple to $2 d$ 0-branes and $d(D-4)$-branes. The scalars from $b_{\mu \nu}$ only appear through their derivatives and so are axion-like in that there is a classical symmetry under which they are shifted by constants. Each of these couples to a $(D-3)$-brane. There are also $\mathrm{RR}(p+1)$-form gauge fields for various values of $p$ coupling to $p$-branes and $\tilde{p}$-branes, and $\mathrm{RR}$ axionic scalars coupling to $(D-3)$-branes (this can be thought of as the case $p=-1$, for which there is only a magnetic brane).

The mass formula (4.5) then gives the following brane scan for the NS-NS branes $(\gamma=2)$ :

\begin{tabular}{|c|c|c|c|c|c|}
\hline Value of $p$ & 1 & $1=D-5$ & 0 & $0=D-4$ & $-1=D-3$ \\
\hline$\rho_{p}=\epsilon_{p} / p+1$ & 0 & $2 / \mathrm{D}-4$ & 0 & $2 / \mathrm{D}-3$ & $2 / \mathrm{D}-2$ \\
\hline$M_{p} / T_{p}$ in string metric & 1 & $g^{-2}$ & 1 & $g^{-2}$ & $g^{-2}$ \\
\hline$M_{p} / T_{p}$ in general metric & $g^{-2 \alpha}$ & $g^{-(2+(D-4) \alpha)}$ & $g^{-\alpha}$ & $g^{-(2+(D-3) \alpha)}$ & $g^{-(2+(D-2) \alpha)}$ \\
\hline
\end{tabular}

Table 4 Type II NS-NS Brane-Scan in D Dimensions.

while the RR branes $(\gamma=0)$ have the following scan:

\begin{tabular}{|c|c|c|}
\hline & $p$ & $\tilde{p}=D-p-4$ \\
\hline$\rho_{p}=\epsilon_{p} / p+1$ & $1 / p+1$ & $1 / D-p-3=1 / \tilde{p}+1$ \\
\hline$M_{p} / T_{p}$ in string metric & $g^{-1}$ & $g^{-1}$ \\
\hline$M_{p} / T_{p}$ in general metric & $g^{-(1+(p+1) \alpha)}$ & $g^{-(1+(\tilde{p}+1) \alpha)}=g^{-(1+(D-p-3) \alpha)}$ \\
\hline
\end{tabular}

Table 5 Type II RR Brane-Scan in D Dimensions.

The two columns give the electric and magnetic branes respectively for $p \geq 0$, 
while for the axionic branes with $p=-1$, there are no electric branes but there are magnetic $(D-3)$-branes.

In all dimensions, the minimum value of $\rho$ is 0 and is carried by the NS-NS string and $2 d$ 0-branes. By this it is meant that there are $2 d$ 'elementary' $p$-branes carrying just one unit of charge [1], while the full 0-brane spectrum of charges is given by a $2 d$-dimensional lattice preserved by the T-duality group $S O(d, d ; \mathbb{Z})$. These then constitute the perturbative spectrum at weak coupling and are the expected result from a compactified string theory: a string plus $d$ winding modes and $d$ momentum modes.

The maximum value of $\rho$ for $10>D>6$ (and for type IIA in $D=10$ ) is 1 and is carried by the RR 0-branes so that the perturbative states of the strong coupling theory are those of a field theory, which as we shall see in the next section are those of 11-dimensional supergravity compactified on a $(d+1)$-torus with coupling constant corresponding to the inverse volume of the torus, as expected from [3]. For $D \leq 6$, however, the RR 0-branes no longer win over the other branes. In $D=6$, the maximum value of $\rho$ is again 1 and is carried by both the $8 \mathrm{RR} 0$-branes and the magnetic NS-NS string resulting from a 5-brane wrapped around the 4-torus. The perturbative spectrum at strong coupling is then again that of a compactified string theory, consisting of a 6 -dimensional string plus 4 momentum modes and 4 winding modes. This is the expected string-string duality. In $D=5$, the maximum value of $\rho$ is 2 and is carried by the NS-NS magnetic 0-brane coming from wrapping the NS-NS 5-brane round the 5-torus. The strong-coupling perturbative theory is the field theory emerging in the large radius limit of the $D=6$ type II theory compactified on a circle.

Thus above six dimensions the RR 0-branes win out and the resulting dynamics is governed by a field theory, but in less than six dimensions the strong coupling theory is dominated by magnetic NS-NS branes. In the intermediate case of six dimensions, the RR 0-branes and the NS-NS string have equal values of $\rho$ and so are both perturbative. 


\section{Compactified Type II Theories and U Duality}

Consider a supergravity theory in $D<10$ dimensions with at least half the maximal number of supersymmetries (e.g. $N \geq 4$ in $D=4$ ). The scalar fields take values in the coset space $G / G_{c}$ where $G$ is the supergravity duality group of symmetries of the equations of motion and $G_{c}$ is the maximal compact subgroup of $G$. The coupling constants are the scalar expectation values. One of the lessons of U-duality is that the coupling constants are all on the same footing and in principle any of them can be given the preferential treatment usually afforded to the dilaton in string theory. A given coupling constant $g$ is $e^{T}$ where $T$ is a noncompact element of the Lie algebra of $G$ and is associated with a scalar expectation $T=\langle\phi\rangle$ for some $\phi \in G / G_{c}$. We will be interested in the dependence of masses on the coupling $g$ and in particular in the strong coupling limit $g \rightarrow \infty$ and the weak coupling limit $g \rightarrow 0$. Our aim will be to find those states that are in the perturbative spectrum at weak coupling and at strong coupling

The curve $e^{t T}$ parameterised by $t$ defines an $\mathbb{R}^{+}$subgroup of $G$ and induces a maximal embedding

$$
H \times \mathbb{R}^{+} \subset G
$$

for some $H$. Factoring both sides by their maximal compact subgroup induces a decomposition of the moduli space of the form

$$
\frac{G}{G_{c}} \sim \frac{H}{H_{c}} \times \mathbb{R}^{+} \times \mathbb{R}^{M}
$$

where $H_{c}$ is the maximal compact subgroup of $H$ and $\mathbb{R}^{M}$ is a vector space equipped with a representation of $H$ [5]. A boundary of the moduli space is then given by going to infinity in the $\mathbb{R}^{+}$direction, i.e. by taking $t \rightarrow \infty$. It was argued in $[3,5]$ that it is sufficient to consider those subgroups $H$ that can be obtained by removing from the Dynkin diagram of $G$ a single vertex representing a non-compact simple root. In a quantum theory for which the supergravity is a low-energy effective 
action (as in string theory), the classical supergravity duality group is broken to a discrete subgroup $G(\mathbb{Z})$, which is the conjectured U-duality symmetry of the theory $[1]$.

The $n$-form gauge fields of the supergravity theory formulated with respect to the Einstein metric transform as a representation $R_{n}$ of $G$ and this implies that the magnetic $p$-branes with $p=D-n-3$ that couple to these have charges transforming as the $R_{n}$ representation of $G$ while the corresponding electric $p$ branes with $p=n-1$ have charges that transform according to the contragredient representation $R_{n}^{\prime}$. In either case, the embedding (7.1) induces a decomposition of the $G$ representation $R$ :

$$
R \rightarrow \oplus_{i}\left(R_{i}^{H}\right)^{\tilde{\epsilon}_{i}}
$$

where the $R_{i}^{H}$ are representations of $H$ and the $\tilde{\epsilon}_{i}$ are the corresponding $\mathbb{R}^{+}$weights. This implies that the mass per unit $p$-volume of the $p$-branes transforming as the $R_{i}^{H}$ of $H$ have the following $g$-dependence with respect to the Einstein metric $g_{\mu \nu}$ :

$$
M_{p}=g^{-\tilde{\epsilon}_{i}} T_{p}
$$

where the effective tension $T_{p}$ can depend on the other coupling constants in $H / H_{c} \times \mathbb{R}^{M}$. Then for the general metric $e^{2 \tilde{\alpha} \phi} g_{\mu \nu}$, the mass per unit volume becomes

$$
M_{p}=g^{-(p+1)\left(\tilde{\rho}_{i}+\tilde{\alpha}\right)} T_{p}
$$

where

$$
\tilde{\rho}_{i}=\frac{1}{p+1} \tilde{\epsilon}_{i}
$$

The $p$-branes can then be assigned to representations $\left(\mathbf{R}_{\mathbf{i}}^{\mathbf{H}}\right)_{\tilde{\rho}_{i}}^{\tilde{\epsilon}_{i}}$ with $H$ representation $\mathbf{R}_{\mathbf{i}}^{\mathbf{H}}$ and $\mathbb{R}^{+}$weight $\tilde{\epsilon}_{i}$, while $\tilde{\rho}_{i}$ is defined by $\tilde{\rho}_{i}=\tilde{\epsilon}_{i} /(p+1)$. In this way the parameters $\tilde{\rho}$ can be found for all $p$-branes and all choices of $H$. For a given $H$ the $p$-branes corresponding to the maximum and minimum values of $\tilde{\rho}$ are the perturbative states of the strong and weak coupling limits, respectively. 
We shall now examine the consequences of this in a number of examples, finding broad agreement with the conjectures of $[3,5]$. This gives an important check on these conjectures, which were based on the 0-brane spectrum only. It is a nontrivial result that the behaviour of the $p$-brane spectrum supports these conjectures, rather than e.g. the conjectured string/(D-5)-brane duality. Furthermore, this analysis will enable us to treat cases that could not be analysed in $[3,5]$. We shall consider first the maximal supergravity theories in $D$ dimensions, given by compactifying 11-dimensional supergravity on an $11-D$ torus or 10-dimensional type IIA or IIB supergravity a $10-D$ torus, which arise from compactified Mtheory or compactified type II superstrings.

\subsection{Type II in $\mathrm{D}=7$, Duality Group $G=S L(5)$}

The maximal supergravity in 7 dimensions has duality group $S L(5)$ with 5 2-form gauge fields transforming as the $\mathbf{5}$ of $S L(5)$ and 10 1-form gauge fields transforming as the $\mathbf{1 0}^{\prime}$ of $S L(5)$. The 2-forms couple to magnetic 2-branes transforming as a $\mathbf{5}$ and electric 1-branes transforming as a $\mathbf{5}^{\prime}$. The 1-forms couple to magnetic 3-branes transforming as a $\mathbf{1 0}^{\prime}$ and electric 0-branes transforming as a 10. There are two choices of $H, S L(4)$ and $S L(3) \times S L(2)$.

$\underline{\text { The } S L(4) \times \mathbb{R}^{+} \text {Decomposition }}$

Here $S L(4) \sim S O(3,3)$ is the T-duality group of the type II string in $D=7$, so that $g$ can be associated with the string coupling constant of the type II string compactified on $T^{3}$. The decomposition of the relevant $S L(5)$ representations under $S L(4) \times \mathbb{R}^{+}$is

$$
5=1^{4}+4^{-1}, \quad \mathbf{1 0}=4^{3}+6^{-2}
$$

where the exponent is the $\mathbb{R}^{+}$weight $\tilde{\epsilon}$. This gives the following brane-scan 


\begin{tabular}{|c|c|}
\hline 0-branes & $\mathbf{6}_{-2}^{-2}+\mathbf{4}_{3}^{3}$ \\
\hline 1-branes & $\mathbf{1}_{-2}^{-4}+\mathbf{4}_{1 / 2}^{\prime 1}$ \\
\hline 2-branes & $\mathbf{1}_{4 / 3}^{4}+\mathbf{4}_{-1 / 3}^{-1}$ \\
\hline 3-branes & $\mathbf{4}_{-3 / 4}^{\prime-3}+\mathbf{6}_{1 / 2}^{\prime 2}$ \\
\hline
\end{tabular}

Table $6 S L(4) \times \mathbb{R}^{+}$Decomposition of $D=7$ Type II Brane-Scan.

(Recall that the $p$-branes are assigned to representations $\left(\mathbf{R}_{\mathbf{i}}^{\mathbf{H}}\right)_{\tilde{\rho}_{i}}^{\tilde{\epsilon}_{i}}$ with $H=S L(4)$ representation $\mathbf{R}_{\mathbf{i}}^{\mathbf{H}}, \mathbb{R}^{+}$weight $\tilde{\epsilon}_{i}$ and value of $\tilde{\rho}_{i}=\tilde{\epsilon}_{i} /(p+1)$.) The minimum value of $\tilde{\rho}$ is $\tilde{\rho}_{\text {min }}=-2$ and is carried by the string and six 0 -branes that transform as a singlet and a $\mathbf{6}^{\prime}$ respectively under the T-duality group $S O(3,3)$. These are the perturbative states of the weakly coupled $D=7$ type II string - the fundamental string of the $D=10$ type II theory gives a string plus 3 momentum mode 0-branes and 3 winding mode 0 -branes on compactification on $T^{3}$. The coupling constant $g$ is the string coupling and $\phi$ is the dilaton. Thus the correct treatment of the $D=7$ supergravity theory as a perturbation theory in $g$ is as a string theory, as expected.

The maximum value of $\tilde{\rho}$ is $\tilde{\rho}_{\min }=3$ and is carried by four 0 -branes, fitting into $4 D=7$ supergravity multiplets that transform as a $4^{\prime}$ under $S L(4)$. This gives the same set of perturbative states that emerged in section 4 from 11-dimensional supergravity compactified on a 4-torus of volume $V$ in the large $V$ limit, where the group $S L(4)$ acted as the mapping class group of the $T^{4}$. This is consistent with the proposal [3] that the theory that emerges in the strong coupling limit of the seven dimensional type II string theory is an 11-dimensional theory compactified on $T^{4}$. Moreover, we learn that the perturbative theory that emerges is a particle theory, not a supermembrane theory.

$\underline{\text { The } S L(2) \times S L(3) \times \mathbb{R}^{+} \text {Decomposition }}$

The relevant $S L(5)$ representations decompose under $S L(2) \times S L(3) \times \mathbb{R}^{+}$as

$$
\mathbf{5}=(\mathbf{2}, \mathbf{1})^{3}+(\mathbf{1}, \mathbf{3})^{-2}, \quad \mathbf{1 0}=(\mathbf{1}, \mathbf{1})^{6}+\left(\mathbf{1}, \mathbf{3}^{\prime}\right)^{-4}+(\mathbf{2}, \mathbf{3})^{1}
$$


This leads to the following brane-scan

\begin{tabular}{|l|c|}
\hline 0-branes & $(\mathbf{1}, \mathbf{1})_{6}^{6}+\left(\mathbf{1}, \mathbf{3}^{\prime}\right)_{-4}^{-4}+(\mathbf{2}, \mathbf{3})_{1}^{1}$ \\
\hline 1-branes & $\left(\mathbf{2}^{\prime}, \mathbf{1}\right)_{-3 / 2}^{-3}+\left(\mathbf{1}, \mathbf{3}^{\prime}\right)_{1}^{2}$ \\
\hline 2-branes & $(\mathbf{2}, \mathbf{1})_{1}^{3}+(\mathbf{1}, \mathbf{3})_{-2 / 3}^{-2}$ \\
\hline 3-branes & $(\mathbf{1}, \mathbf{1})_{-3 / 2}^{-6}+(\mathbf{1}, \mathbf{3})_{1}^{4}+\left(\mathbf{2}^{\prime}, \mathbf{3}^{\prime}\right)_{-1 / 4}^{-1}$ \\
\hline
\end{tabular}

Table $7 S L(2) \times S L(3) \times \mathbb{R}^{+}$Decomposition of $D=7$ Type II Brane-Scan.

The minimum value of $\tilde{\rho}$ is -4 and is carried by 0 -branes in the $\left(\mathbf{1}, \mathbf{3}^{\prime}\right)$ representation of $S L(2) \times S L(3)$ and is the same perturbative spectrum that arises from type IIB supergravity compactified on $T^{3}$ in the limit of large torus volume, with $S L(2)$ the type IIB duality group and $S L(3)$ the $T^{3}$ mapping class group. If we consider the type IIB superstring compactified on $T^{3}$, the low energy effective effective action is the seven dimensional supergravity theory under consideration, and the spectrum of $p$-branes is the same. Thus this perturbation theory of the type IIB superstring gives a field theory, not a string theory.

The maximum value of $\tilde{\rho}$ is 6 and is carried by 0 -branes in the $(\mathbf{1}, \mathbf{1})$ representation of $S L(2) \times S L(3)$ and is the same perturbative spectrum that arises from 8-dimensional supergravity or $D=8$ type II superstring compactified on a circle in the large radius limit.

These results are in agreement with [3], but clarify the nature of the compactified theory. Different perturbative theories emerge with different choices of perturbation parameter, corresponding to different choices of $H$. Choosing $H=S L(4)$ as in the previous subsection gives a perturbative string theory in which the perturbation parameter is the string coupling, related to the dilaton in the usual way, while choosing $H=S L(2) \times S L(3)$ corresponds to perturbing in the inverse volume of the torus, and the perturbative theory that emerges is a field theory, not a string theory. 


\subsection{Type II in $\mathrm{D}=6$, Duality Group $G=S O(5,5)$}

The maximal supergravity in 7 dimensions has duality group $S O(5,5)$ with 16 1-form gauge fields transforming as the spinor $\mathbf{1 6}$ of $S O(5,5)$ and 5 2-form gauge fields. The 53 -form field strengths $H^{a}$, together with the dual field strengths ${ }^{\star}$ form a 10 -vector transforming as a $\mathbf{1 0}$ of of $S O(5,5)$. There are then 160 -branes transforming as a $\mathbf{1 6} \mathbf{6}^{\prime}, 16$ 2-branes transforming as a $\mathbf{1 6}$, and 10 strings transforming as a 10. There are a number of choices of $H$ to consider.

$\underline{\text { The } S O(4,4) \times \mathbb{R}^{+} \text {Decomposition }}$

Here $S O(4,4)$ is the T-duality group of the type II string in $D=6$, so that $g$ can be associated with the string coupling constant of the type II string compactified on $T^{3}$. Under $S O(4,4) \times \mathbb{R}^{+}$,

$$
\mathbf{1 6}=\mathbf{8}_{\mathrm{s}}{ }^{1}+\mathbf{8}_{\mathrm{c}}{ }^{-1}, \quad \mathbf{1 0}=\mathbf{1}^{2}+\mathbf{1}^{-2}+\mathbf{8}_{\mathrm{v}}{ }^{0}
$$

This gives the following brane-scan

\begin{tabular}{|c|c|}
\hline 0-branes & $\mathbf{8}_{\mathbf{s} 1}^{1}+\mathbf{8}_{\mathbf{c}_{-1}^{-1}}^{-1}$ \\
\hline 1-branes & $\mathbf{1}_{1}^{2}+\mathbf{1}_{-1}^{-2}+\mathbf{8}_{\mathbf{v} 0}^{0}$ \\
\hline 2-branes & $\mathbf{8}_{\mathbf{c}_{1 / 3}^{1}+\mathbf{8}_{\mathbf{s}_{-1}}^{-1}}^{1}$ \\
\hline
\end{tabular}

Table $8 S O(4,4) \times \mathbb{R}^{+}$Decomposition of $D=6$ Type II Brane-Scan.

The maximum value of $\tilde{\rho}$ is $\tilde{\rho}_{\max }=1$ and is carried by the string and 80 branes that transform as a singlet and an 8 respectively under the T-duality group $\mathrm{SO}(4,4)$. These are the perturbative states of the weakly coupled $D=6$ type II string - the fundamental string of the $D=10$ type II theory gives a string plus 4 momentum mode 0-branes and 4 winding mode 0-branes on compactification on

\footnotetext{
$\star$ The dual field strength is given by the Hodge dual of the variation of the action with respect to $H^{a}, \tilde{H}_{a}=*\left(\delta S / \delta H^{a}\right)$ transforming as the $\mathbf{5}^{\prime}$. Thus the Bianchi identities are $d H^{a}=0$ and the field equations are $d \tilde{H}_{a}=0$.
} 
$T^{4}$. The minimum value of $\tilde{\rho}$ is $\tilde{\rho}_{\text {min }}=-1$ and is again carried by a string and 80 -branes, reflecting the self-duality of the theory that is predicted by U-duality. Both strong and weak coupling limits give the same perturbative string theory, which is that of a compactified type II string.

$\underline{\text { The } S L(5) \times \mathbb{R}^{+} \text {Decomposition }}$

The decomposition of the relevant representations under $S L(5) \times \mathbb{R}^{+}$is

$$
16=1^{-5}+5^{\prime 3}+10^{-1}, \quad 10=5^{2}+5^{\prime-2}
$$

The resulting brane-scan is

\begin{tabular}{|c|c|}
\hline 0-branes & $\mathbf{1}_{-5}^{-5}+\mathbf{5}_{3}^{\prime 3}+\mathbf{1 0}_{-1}^{-1}$ \\
\hline 1-branes & $\mathbf{5}_{1}^{2}+\mathbf{5}_{-1}^{\prime-2}$ \\
\hline 2-branes & $\mathbf{1}_{5 / 3}^{5}+\mathbf{5}_{-1}^{-3}+\mathbf{1 0}_{1 / 3}^{\prime 1}$ \\
\hline
\end{tabular}

Table $9 S L(5) \times \mathbb{R}^{+}$Decomposition of $D=6$ Type II Brane-Scan.

The maximum value of $\tilde{\rho}$ is 3 and is carried by 5 0-branes, fitting into $5 D=6$ supergravity multiplets that transform as a $5^{\prime}$ under $S L(5)$. This gives the same set of perturbative states that emerged in section 4 from 11-dimensional supergravity compactified on a 5 -torus of volume $V$ in the large $V$ limit, where the group $S L(5)$ acted as the mapping class group of the $T^{5}$.

The minimum value of $\tilde{\rho}$ is -5 and is carried by the $S L(5)$ singlet 0-brane, and the perturbative theory is that of 7-dimensional type II supergravity compactified on a circle, in the large radius limit.

The $S L(4) \times S L(2) \times \mathbb{R}^{+}$Decomposition

The relevant $S O(5,5)$ representations decompose under $S L(4) \times S L(2) \times \mathbb{R}^{+}$ as

$$
\begin{aligned}
& \mathbf{1 6}=(\mathbf{4}, \mathbf{1})^{1}+(\mathbf{4}, \mathbf{1})^{-1}+\left(\mathbf{4}^{\prime}, \mathbf{2}\right)^{0} \\
& \mathbf{1 0}=(\mathbf{1}, \mathbf{2})^{1}+(\mathbf{1}, \mathbf{2})^{-1}+(\mathbf{6}, \mathbf{1})^{0}
\end{aligned}
$$


Both the maximum and minimum values of $\tilde{\rho}( \pm 1)$ are carried by 0-branes in the $(\mathbf{4}, \mathbf{1})$ representation of $S L(4) \times S L(2)$, so that the theory is self-dual and the same perturbative theory as arises in both these limits also emerges from the type IIB theory compactified on $T^{4}$ in the limit of large or small torus volume, with $S L(2)$ the type IIB duality group and $S L(4)$ the $T^{4}$ mapping class group.

$\underline{\text { The } S L(2) \times S L(2) \times S L(3) \times \mathbb{R}^{+} \text {Decomposition }}$

The relevant $S O(5,5)$ representations decompose under $S L(2) \times S L(2) \times$ $S L(3) \times \mathbb{R}^{+}$as

$$
\begin{aligned}
& \mathbf{1 6}=(\mathbf{2}, \mathbf{1}, \mathbf{1})^{3}+(\mathbf{2}, \mathbf{1}, \mathbf{3})^{-1}+(\mathbf{1}, \mathbf{2}, \mathbf{1})^{-3}+\left(\mathbf{1}, \mathbf{2}, \mathbf{3}^{\prime}\right)^{1} \\
& \mathbf{1 0}=(\mathbf{2}, \mathbf{2}, \mathbf{1})^{0}+(\mathbf{1}, \mathbf{1}, \mathbf{3})^{2}+(\mathbf{1}, \mathbf{1}, \mathbf{3})^{-2}
\end{aligned}
$$

The maximum and minimum values of $\tilde{\rho}( \pm 3)$ are carried by 0 -branes in the $(\mathbf{2}, \mathbf{1}, \mathbf{1})$ and $(\mathbf{1}, \mathbf{2}, \mathbf{1})$ representations of $S L(2) \times S L(2) \times S L(3)$ and these correspond to the $D=8$ theory compactified on $T^{2}$ in the limits of large volume and large values of the complex structure modulus, respectively.

\subsection{Type II in $\mathrm{D}=5$, Duality Group $G=E_{6}$}

The maximal supergravity in 6 dimensions has duality group $E_{6(6)}$ with 27 1form gauge fields transforming as the $\mathbf{2 7}$ of $E_{6}$. These couple to 27 electric 0 -branes and 27 magnetic strings, transforming as a $\mathbf{2 7 ^ { \prime }}$ and $\mathbf{2 7}$ respectively.

The $S O(5,5) \times \mathbb{R}^{+}$Decomposition

The $\mathbf{2 7}$ decomposes under $S O(5,5) \times \mathbb{R}^{+}$as

$$
27=1^{4}+10^{-2}+16^{1}
$$

The minimum value of $\tilde{\rho}$ is -2 and is carried by a string and 100 -branes in the (10) of the T-duality group $S O(5,5)$, corresponding to the weakly coupled $D=5$ type II string theory. The maximum value of $\tilde{\rho}$ is 4 and is carried by a single 0 -brane, corresponding to the large radius limit of the $D=6$ type II theory compactified on a circle. 
The $S L(6) \times \mathbb{R}^{+}$Decomposition

The $\mathbf{2 7}$ decomposes under $S L(6) \times \mathbb{R}^{+}$as

$$
27=6^{1}+6^{\prime-1}+15^{0}
$$

The maximum and minimum values of $\tilde{\rho}( \pm 1)$ are each carried by 60 -branes in the $\mathbf{6}^{\prime}$ representation of $S L(6)$ so that the theory is self-dual in this direction in coupling constant space and either limit corresponds to the $D=11$ theory compactified on $T^{6}$ in the limits of large or small torus volume.

$\underline{\text { The } S L(2) \times S L(5) \times \mathbb{R}^{+} \text {Decomposition }}$

The $\mathbf{2 7}$ decomposes under $S L(2) \times S L(5) \times \mathbb{R}^{+}$as

$$
\mathbf{2 7}=(\mathbf{2}, \mathbf{1})^{5}+\left(\mathbf{2}, \mathbf{5}^{\prime}\right)^{-1}+(\mathbf{1}, \mathbf{5})^{-4}+(\mathbf{1}, \mathbf{1 0})^{2}
$$

The maximum value of $\tilde{\rho}$ is 5 and is carried by 0 -branes in the $(\mathbf{2}, \mathbf{1})$ representation of $S L(2) \times S L(5)$ and corresponds to the $D=7$ theory compactified on $T^{2}$ in the limit of large torus volume. The minimum value is $\tilde{\rho}=-4$ and is carried by 0 -branes in the $(\mathbf{1}, \mathbf{5})$ representation of $S L(2) \times S L(5)$ and corresponds to the $D=10$ type IIB theory compactified on $T^{5}$.

The $S L(3) \times S L(3) \times S L(2) \times \mathbb{R}^{+}$Decomposition

The $\mathbf{2 7}$ decomposes under $S L(2) \times S L(5) \times \mathbb{R}^{+}$as

$$
\mathbf{2 7}=\left(\mathbf{3}, \mathbf{3}^{\prime}, \mathbf{1}\right)^{0}+(\mathbf{3}, \mathbf{1}, \mathbf{1})^{-2}+(\mathbf{3}, \mathbf{1}, \mathbf{2})^{1}+\left(\mathbf{1}, \mathbf{3}^{\prime}, \mathbf{1}\right)^{2}+\left(\mathbf{1}, \mathbf{3}^{\prime}, \mathbf{2}^{\prime}\right)^{-1}
$$

The maximum and minimum values of $\tilde{\rho}( \pm 2)$ are carried by 0 -branes in the $(\mathbf{3}, \mathbf{1}, \mathbf{1})$ and $\left(\mathbf{1}, \mathbf{3}^{\prime}, \mathbf{1}\right)$ representations and these correspond to the $D=8$ theory compactified on $T^{3}$. 


\section{Heterotic Theories and $K_{3}$ Compactifications}

In the previous sections we have considered theories with maximal supersymmetry whose low energy supergravity effective action comes from toroidally compactifying $N=2$ supergravity theories from ten dimensions. In this section, we will turn to theories with half the maximal number of supersymmetries $-N=1$ in $D=10$ or $N=4$ in $D=4$ - which can result from toroidal compactification of heterotic or type I theories, or from compactification of type II theories on $K_{3} \times T^{n}$. In each case, the brane-scan is again determined by low-energy supersymmetry, so we shall start from the brane-scan of the supergravity theory, find which are the perturbative states for the various coupling constants, and then seek an interpretation of the resulting perturbation theory.

In $D>4$ dimensions, the half-maximal supergravity theory coupled to $n$ abelian vector multiplets (resulting from toroidal compactification of $N=1$ theories in $D=10$ ) has scalar fields lying in the coset space

$$
\frac{O(d, d+m)}{O(d) \times O(d+m)} \times \mathbb{R}^{+}
$$

where $d=10-D$ and $m=n-d$ and has $2 d+m$ vector fields transforming as the vector representation of the duality group $O(d, d+m)$. For the heterotic string, $m=16, O(d, d+16)$ is the T-duality group and the dilaton takes values in the $\mathbb{R}^{+}$factor in (8.1). The 2-form gauge field couples to a string and a $(D-5)$ brane, while the vector fields couple to 0-branes whose charges take values in a $2 d+m$-dimensional lattice and $(D-4)$-branes whose charges take values in the $2 d+m$-dimensional dual lattice. In $D \leq 8$ dimensions, the ten-dimensional 2 form gives rise to $d(d-1) / 2$ scalars that couple to magnetic $(D-3)$-branes. As described in section 5.3, there may also be other branes, possibly related to type I strings. However, in $D \leq 7$ the branes considered are precisely those that come from compactifying 11-dimensional supergravity or M-theory on $K_{3} \times T^{N}$ (where $N=7-D)$ and as yet no extra exotic branes are suspected in these theories. We 
shall start by studying the known branes and investigating their dependence on the various coupling constants in (8.1).

\subsection{The Heterotic Dilaton}

In this section, we shall investigate perturbation theory in the coupling constant $g$ in the $\mathbb{R}^{+}$factor of (8.1), which corresponds to the heterotic string dilaton $\Phi$, $g=e^{\langle\Phi\rangle}$. The brane scan is

\begin{tabular}{|c|c|c|c|c|}
\hline Value of $p$ & 1 & $1=D-5$ & 0 & $0=D-4$ \\
\hline$O(d, d+m)$ Representation & $\mathbf{1}$ & $\mathbf{1}$ & $2 d+m$ & $2 d+m$ \\
\hline$\rho_{p}=\epsilon_{p} / p+1$ & 0 & $2 / \mathrm{D}-4$ & 0 & $2 / \mathrm{D}-3$ \\
\hline$M_{p} / T_{p}$ in string metric & 1 & $g^{-2}$ & 1 & $g^{-2}$ \\
\hline$M_{p} / T_{p}$ in general metric & $g^{-2 \alpha}$ & $g^{-(2+(D-4) \alpha)}$ & $g^{-\alpha}$ & $g^{-(2+(D-3) \alpha)}$ \\
\hline
\end{tabular}

Table 10 Heterotic Brane-Scan in D Dimensions.

In addition there are $(D-3)$-branes that couple to certain scalars, such as those that arise from the 2 -form gauge field on compactification and for which $\rho_{p}=2 / D-2$. The perturbative states at weak coupling $(\rho=0)$ are, as expected, a string plus $2 d+m$ 0-branes (generating a $2 d+m$ dimensional lattice of supermultiplets). The latter consist of $d$ momentum and $d$ winding modes from a string on a $d$-torus, plus the $m$ heterotic winding/momentum modes from a left-moving string on an $m$-torus; for the heterotic string, $m=16$. Of these $2 d+m 0$-branes and 6 -branes, only $d$ 0-branes and $d$ 6-branes saturate a Bogomolnyi bound and preserve half the supersymmetry; the others are not supersymmetric. For strong coupling, the maximum value of $\rho$ in the table is $2 / D-4$ and is carried by the $(D-5)$-brane. If there are no other branes in the spectrum with higher values of $\rho$, then this would imply that the strong-coupling perturbative spectrum consists of a $(D-5)$-brane. In section 5.3, however, reasons were given for expecting new type I branes with a higher value of $\rho$ in $D=10$, and the conjectured type-I/heterotic duality in $D \geq 8$ dimensions [3] suggests that the highest value of $\rho$ should be carried by such branes 
in $D=8,9,10$. We shall therefore concentrate on $D<8$ dimensions here.

In $D=6$, the strong coupling perturbative spectrum consists of the $(D-$ 5)-brane, which in this case is a string. There are no 0-branes, so this doesn't correspond to the spectrum of a toroidally compactified heterotic string, but is precisely the weak-coupling perturbative spectrum of the type IIA string theory compactified on $K_{3}$, with type IIA coupling constant $g^{\prime}=1 / g$. Thus the strongly coupled heterotic string in $D=6$ is described by a weakly coupled type IIA string theory compactified on $K_{3}$, as expected $[1,3]$.

Consider an 11-dimensional theory (supergravity or M-theory) compactified on $K_{3} \times S^{1}$. Large and small values of $g$ correspond to large and small values of the radius of the circle, respectively. Thus, we learn that in the large radius limit, the perturbation theory arising from expanding in the inverse radius is a string theory, as expected from the corresponding results for compactifying from 11 dimensions to 10 on $S^{1}$.

In $D=5$ dimensions, the strong coupling spectrum consists of a single 0-brane supermultiplet, and this is described by the supergravity theory resulting from type IIB compactified on $K_{3} \times S^{1}$, with the $g \rightarrow \infty$ limit corresponding to that in which the radius of the circle becomes infinite [3].

In $D=7$ dimensions, however, the BPS-saturated brane with the highest value of $\rho$ is a supermembrane. Precisely the same brane-scan arises from 11-dimensional supergravity compactified on a $K_{3}$ with radius $g^{1 / 3}$, and it was suggested in [3] that the strongly coupled $D=7$ heterotic string should correspond to $K_{3}$-compactified 11-dimensional supergravity in the large radius limit. However, if this were the case, then one would expect the perturbative spectrum to consist of 0-branes instead of membranes; this was certainly the way things worked so far. There seem to be two possibilities. The first is that this is indeed the correct perturbative spectrum and that the large $g$ behaviour is described by a perturbative supermembrane theory in $D=7$, as considered in [41]; it is remarkable that in contrast to the case of 11dimensions there is a coupling constant and such a theory could exist. The second 
possibility is that there are other non-perturbative states in the spectrum that govern the strong coupling, perhaps corresponding to branes with higher values of $\rho$. This would be analogous to what happened in the type IIA theories: the extra states needed to 'beat' the $(D-5)$-branes in $D \geq 7$ arose from the $\mathrm{RR}$ sector and the strong coupling theory was described in terms of RR 0-branes. This possibility seems the more likely, and might work as follows. ${ }^{\star}$ 11-dimensional supergravity compactified on $K_{3}$ has Kaluza-Klein scalars in its spectrum that become massless in the large volume limit. They are not BPS-saturated and so, although they should be in the pertrurbative spectrum at large volume, they should not be expected to persist for other values of the coupling. With the choice $\alpha=-1$, these KaluzaKlein 0-branes have $M / T \sim 1$, while the string, membrane, heterotic 0-branes and 3-branes have $M / T$ given by $g^{2}, g, g, g^{2}$ respectively. Thus if the heterotic string in $D=7$ has such non-BPS 0-brane states that are metastable at strong coupling but which may not continue to states at weak coupling, then these would be the perturbative states at strong coupling and this is what must happen if the strong coupling limit is to be $K_{3}$-compactified 11-dimensional supergravity.

\subsection{Other Coupling Constants}

We now turn to the coupling constants in the $O(d, d+m) / O(d) \times O(d+m)$ factor in (8.1). From [5], the distinct possibilities are labelled by an integer $n$ and constitute going to a boundary in the moduli space in which

$$
\frac{O(d, d+m)}{O(d) \times O(d+m)} \rightarrow \frac{O(d-n, d-n+m)}{O(d-n) \times O(d-n+m)} \times \frac{S L(n, \mathbb{R})}{S O(n)} \times \mathbb{R}^{+} \times \mathbb{R}^{M}
$$

for some $M$. We will be interested in the dependence on the coupling constant $g$ corresponding to the $\mathbb{R}^{+}$factor in (8.2). The 2 -form is neutral under $O(d, d+m)$ and so the corresponding string and $(D-5)$-form will not couple to this coupling

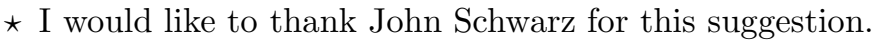


constant. The vector fields transform as the fundamental representation, which decomposes under

$$
O(d, d+m) \rightarrow O(d-n, d-n+m) \times S L(n, \mathbb{R}) \times \mathbb{R}^{+}
$$

as

$$
\mathbf{2 d}+\mathbf{m} \rightarrow(\mathbf{2}(\mathbf{d}-\mathbf{n})+\mathbf{m}, \mathbf{1})^{0}+(\mathbf{1}, \mathbf{n})^{1}+\left(\mathbf{1}, \mathbf{n}^{\prime}\right)^{-1}
$$

The resulting brane-scan for the 0,1 branes and their magnetic duals is

\begin{tabular}{|c|c|}
\hline 0-branes & $(2(d-n)+m, 1)_{0}^{0}+(1, n)_{1}^{1}+\left(1, n^{\prime}\right)_{-1}^{-1}$ \\
\hline 1-branes & $1_{0}^{0}$ \\
\hline (D-5)-branes & $1_{0}^{0}$ \\
\hline (D-4)-branes & $(2(d-n)+m, 1)_{0}^{0}+\left(1, n^{\prime}\right)_{1 / D-3}^{1}+(1, n)_{1 / D-3}^{-1}$ \\
\hline
\end{tabular}

Table 11 Heterotic Brane-Scan.

In $D \geq 4$ the maximum and minimum values of $\rho$ are \pm 1 and are carried by $n$ 0-branes, so that the perturbative spectrum for both $g$ and $1 / g$ consists of $n$ 0-brane multiplets in $D>4$ and $2 n$ 0-brane multiplets in $D=4$.

We now turn to the interpretation of these results, using the discussion of [5]. The factor $\frac{S L(n, R)}{S O(n)}$ in (8.2) is the moduli space of fixed-volume metrics on an $n$ torus. From earlier discussions, the perturbative spectrum of a supergravity theory compactified on $T^{n}$ with respect to the coupling constant representing the volume of $T^{n}$ consists of $n$ 0-brane multiplets in the lower dimension, and this is consistent with the results just found. The factor

$$
\frac{O(d-n, d-n+m)}{O(d-n) \times O(d-n+m)}
$$

in (8.2) can be interpreted as a heterotic string moduli space or as the moduli space 
of $K_{3}$ or some squashed $K_{3}$ for certain values of $d, n, m$. First,

$$
\frac{O(M, M+16)}{O(M) \times O(M+16)}
$$

is the moduli space of heterotic strings in $10-M$ dimensions. Next

$$
\frac{O(3,19)}{O(3) \times O(19)}
$$

is the Teichmüller space of Ricci-flat metrics of fixed volume on $K_{3}$ while

$$
\frac{O(4,20)}{O(4) \times O(20)}
$$

is the moduli space of conformal field theories on $K_{3}$ [42]. The spaces

$$
\frac{O(3-n, 19-n)}{O(3-n) \times O(19-n)}
$$

for $n=1,2,3$ were identified in [5] as the moduli spaces of certain degenerations of $K_{3}$ down to $4-n$ dimensions. It will be convenient to denote the $m=4-n$ dimensional squashed $K_{3}$ as $\Xi^{m}$, with $\Xi^{4}=K_{3}$. There are in fact two distinct 1-dimensional degenerations [5], which we will denote $\Xi_{1}^{1}$ and $\Xi_{2}^{1}$. It was argued in [5] that taking the strong coupling limit in certain directions in coupling constant space corresponds to going to boundaries of the $K_{3}$ moduli space in which $K_{3}$ degenerates to one of the $\Xi^{m}$. As in [5], it will be assumed here that taking this limit makes sense and corresponds to a compactification of M-theory on the $\Xi^{m}$.

$\underline{D=6}$

Consider the case of the six-dimensional theory with scalar coset space

$$
\frac{O(4,20)}{O(4) \times O(20)} \times \mathbb{R}^{+}
$$

which arises from the toroidally compactified heterotic string, type IIA compactified on $K_{3}$ and the 11-dimensional theory compactified on $K_{3} \times S^{1}$. For the 
coupling constant in the $\mathbb{R}^{+}$factor, the weak coupling theory is the perturbative heterotic string and the strong coupling is described by the perturbative type IIA string, as discussed in section 8.1.

Consider first the degeneration

$$
\frac{O(4,20)}{O(4) \times O(20)} \rightarrow \frac{O(3,19)}{O(3) \times O(19)} \times \mathbb{R}^{+} \times \mathbb{R}^{22}
$$

The charged perturbative spectrum in $g$ at weak coupling and in $1 / g$ at strong coupling both consist of a single six-dimensional 0-brane supermultiplet, so that both the weak and strong coupling limits are field theories. The limit $g \rightarrow \infty$ for the coupling constant $g$ corresponding to the $\mathbb{R}^{+}$factor in (8.5) corresponds, for the heterotic string, to a boundary of the $T^{4}$ moduli space in which one of the circles in $T^{4}$ becomes large, so that in the limit the theory decompactifies to the 7-dimensional heterotic string, which has moduli space

$$
\frac{O(3,19)}{O(3) \times O(19)} \times \mathbb{R}^{+}
$$

The limit $g \rightarrow 0$ corresponds to one of the circles becoming small, but this is related by T-duality to the large radius limit of the same theory, so that the theory is selfdual and the weak and strong coupling limits (with respect to $g$ ) define equivalent theories. In the limit $g \rightarrow \infty$ (or $g \rightarrow 0$ ), one recovers 7 -dimensional Lorentz invariance.

Consider now the interpretation of these limits in terms of the 11-dimensional M theory. The limits $g \rightarrow 0$ and $g \rightarrow \infty$ correspond to boundaries of the moduli space of compactifications of the 11-dimensional theory on $K_{3} \times S^{1}$ which lead to 7-dimensional theories and so must be the ones in which the circle becomes large or small. The large radius limit decompactifies to the 7 -dimensional theory resulting from compactifying from 11-dimensions on $K_{3}$, which is expected to correspond to the 7-dimensional heterotic string [3]. We also learn that the small radius limit is equivalent to the large radius limit (using the heterotic equivalence), so that 
the 11-dimensional M-theory must also exhibit some form of T-duality. Thus the perturbation theory of the $D=6$ heterotic string with respect to the coupling constant that vanishes when the theory decompactifies to $D=7$ is described by the field theory arising from compactifying the 11-dimensional theory on $K_{3} \times S^{1}$ and expanding in the inverse circle radius, as expected from [3].

Similar remarks apply to other degenerations. For

$$
\frac{O(4,20)}{O(4) \times O(20)} \rightarrow \frac{O(2,18)}{O(2) \times O(18)} \times \frac{S L(2, \mathbb{R})}{U(1)} \times \mathbb{R}^{+} \times \mathbb{R}^{41}
$$

we obtain two 0-brane supermultiplets (transforming as a $\mathbf{2}$ of $S L(2, \mathbb{R})$ ) as the perturbative spectrum both at weak and strong coupling for the coupling constant corresponding to the $\mathbb{R}^{+}$factor in (8.6). This corresponds to (i) the 8-dimensional heterotic string on $T^{2}$ and (ii) the 11-dimensional theory on $\Xi^{3} \times T^{2}$, assuming this limit makes sense [5]. In each case $g$ is related to the volume of the $T^{2}$, so that $g \rightarrow \infty$ corresponds to decompactification to 8-dimensions, while the $g \rightarrow 0$ limit defines an equivalent theory, related by T-duality. For

$$
\frac{O(4,20)}{O(4) \times O(20)} \rightarrow \frac{O(1,17)}{O(17)} \times \frac{S L(3, \mathbb{R})}{S O(3)} \times \mathbb{R}^{+} \times \mathbb{R}^{57}
$$

we obtain three 0-brane supermultiplets as the perturbative spectrum both at weak and strong coupling. This corresponds to (i) the 9-dimensional heterotic string on $T^{3}$ and (ii) the 11-dimensional theory on $\Xi^{2} \times T^{3}$. Finally, for

$$
\frac{O(4,20)}{O(4) \times O(20)} \rightarrow \frac{S L(4, \mathbb{R})}{S O(4)} \times \mathbb{R}^{+} \times \mathbb{R}^{70}
$$

we obtain four 0-brane supermultiplets as the perturbative spectrum both at weak and strong coupling. This corresponds to (i) the 10-dimensional heterotic string on $T^{4}$ (ii) the 11-dimensional theory on $\Xi^{1} \times T^{4}$.

Similar results apply in other dimensions $D \leq 6$ (cf. [5]) with a perturbative field theory emerging in each case when expanding in couplings corresponding 
to scalars other than the heterotic dilaton. The results are consistent with the conjecture that the heterotic string in $D$ dimensions is equivalent to 11-dimensional M-theory compactified on $\Xi^{11-D}$ and to the type IIA string compactified on $\Xi^{10-D}$, with $\Xi^{n}$ defined to be $K_{3} \times T^{n-4}$ for $n \geq 4$. This gives the following table.

\begin{tabular}{|c|c|c|}
\hline Dimension $D$ & Symmetry Group G & M-Theory Compactified on: \\
\hline 10 & $O(16)$ & $\Xi^{1}$ \\
\hline 9 & $O(1,17)$ & $\Xi^{2}$ \\
\hline 8 & $O(2,18)$ & $\Xi^{3}$ \\
\hline 7 & $O(3,19)$ & $K_{3}$ \\
\hline 6 & $O(4,20)$ & $K_{3} \times T^{1}$ \\
\hline 5 & $O(5,21)$ & $K_{3} \times T^{2}$ \\
\hline 4 & $O(6,22)$ & $K_{3} \times T^{3}$ \\
\hline
\end{tabular}

Table 12 Conjectured duals of $D$-dimensional heterotic string from compactifying M-theory.

There is considerable evidence supporting the relations involving $K_{3}$ or $K_{3} \times T^{n}$ $[1,3,5,4]$ but those involving the degenerations of $K_{3}[5]$ are more speculative and deserve further investigation.

It is interesting to speculate on the relation of these conjectures to those of Horava and Witten [26] that the $E_{8} \times E_{8}$ heterotic string in $D=10$ is related to M-theory compactified from 11 dimensions on the orbifold $S^{1} / \mathbb{Z}_{2}$. Compactifying to $D=7$ on $T^{3}$ (without Wilson lines) gives the $D=7$ heterotic string with gauge group $E_{8} \times E_{8} \times U(1)^{6}$, or M-theory compactified on $T^{3} \times S^{1} / \mathbb{Z}_{2}$. However, the same theory should emerge from the 11-dimensional theory compactified on an orbifold limit of $K_{3}$ at which $E_{8} \times E_{8}$ gauge symmetry emerges [3,4]; it would be interesting to understand further why M-theory compactified on these two orbifolds should give the same theory. This in turn might shed some light on the construction of the $K_{3}$ degenerations $\Xi^{m}$ : M-theory on $\Xi^{m}$ should, at an enhanced symmetry point of the $\Xi^{m}$ moduli space, be equivalent to M-theory on $T^{m-1} \times S^{1} / \mathbb{Z}_{2}$. 


\section{The Chiral Theory in Six Dimensions}

The $\mathrm{N}=2$ supersymmetric theories in six dimensions considered so far have had $(1,1)$ supersymmetry, i.e. the two supersymmetries have opposite chirality. There are also $(2,0)$ theories in $D=6$ with both supersymmetries having the same chirality. For $(2,0)$ supergravity coupled to $n(2,0)$ anti-symmetric tensor multiplets, the scalar fields take values in the coset space

$$
\frac{O(5, n)}{O(5) \times O(n)}
$$

There are $5+n$ anti-symmetric tensor gauge fields $b_{\mu \nu}^{I}(I=1, \ldots, 5+n)$ with corresponding field strengths $H_{\mu \nu \rho}^{I}$ that transform as a vector of $O(5, n)$. The scalars can be represented as a $(5+n) \times(5+n)$ matrix $\mathcal{V}_{I J}$ transforming under rigid $O(5, n)$ from the right and local $O(5) \times O(n)$ from the left. Decomposing the $O(5) \times O(n)$ index into an $O(5)$ index $a$ and an $O(n)$ index $m$, one can form the $O(5, n)$-invariant field strength 3 -forms

$$
G_{a}=\mathcal{V}_{a I} H^{I}, \quad K_{m}=\mathcal{V}_{m I} H^{I}
$$

The 5 field strengths $G_{a}$ are self-dual while the $n$ field strengths $K_{m}$ are anti-selfdual:

$$
G_{a}=* G_{a}, \quad H_{m}=-* H_{m}
$$

Decomposing the $O(n)$ index $m$ into an $O(5)$ index $a$ and an $O(n-5)$ index $u$, $H_{m} \rightarrow\left(H_{a}, H_{u}\right)$ and defining

$$
G_{a}^{ \pm}=G_{a} \pm H_{a}
$$

we obtain 5 unconstrained field strengths $G_{a}^{+}$, their duals $G_{a}^{-}=* G_{a}^{+}$, and $n-5$ anti-self-dual field strengths $H_{u}$. 
The theory with $n=19$ emerges as the low-energy effective field theory for the type IIB superstring compactified on $K_{3}$, with U-duality group $O(5,21 ; \mathbb{Z})[5]$. The $p$-brane spectrum for general $n$ consists of 5 self-dual strings and $n$ anti-self-dual ones, or equivalently 5 ordinary (non-chiral) strings and $n-5$ anti-self-dual ones.

Consider the compactification of this theory to $D=5$. If the type IIB theory is compactified on $K_{3} \times S^{1}$ with circle of radius $R$, it is equivalent to the type IIA theory compactified on $K_{3} \times S^{1}$ with circle of radius $1 / R$, and this is in turn related to the $D=5$ heterotic string [3] and the 11-dimensional theory on $K_{3} \times T^{2}$. However, in $D=6$ the chiral supergravity theory is not related to any of the theories in table 12 .

The general decomposition is

$$
\frac{O(5,21)}{O(5) \times O(21)} \rightarrow \frac{O(5-n, 21-n)}{O(5-n) \times O(21-n)} \times \frac{S L(n, \mathbb{R})}{O(n)} \times \mathbb{R}^{+} \times \mathbb{R}^{M}
$$

where $M=-\frac{3}{2} n^{2}+26 n-\frac{1}{2}$. The $\mathbf{2 6}$ representation of $O(5,21)$ decomposes under

$$
O(5,21) \rightarrow O(5-n, 21-n) \times S L(n, \mathbb{R}) \times \mathbb{R}^{+}
$$

as

$$
\mathbf{2 6} \rightarrow(\mathbf{2 6}-\mathbf{2 n}, \mathbf{1})^{0}+(\mathbf{1}, \mathbf{n})^{1}+\left(\mathbf{1}, \mathbf{n}^{\prime}\right)^{-1}
$$

where the superscript is the $\mathbb{R}^{+}$charge. The corresponding values of $\rho$ for the three terms on the right hand side of (9.4) are $0, \frac{1}{2}$ and $-\frac{1}{2}$, respectively. If there are no metastable non-BPS states with higher or lower values of $\rho$ in the spectrum, then for the coupling constant corresponding to the $\mathbb{R}^{+}$factor in (9.3), the perturbative states at strong coupling are $n$ non-chiral strings (i.e. ordinary strings satisfying no self-duality condition) coupling to $G_{a}^{+}$and transforming as an $\mathbf{n}$ of $S L(n, \mathbb{R})$ while those at weak coupling are $n$ non-chiral strings coupling to $G_{a}^{-}$and transforming as an $\mathbf{n}^{\prime}$ of $S L(n, \mathbb{R})$. 
The case $n=1$ is straightforward to interpret: the decomposition

$$
\frac{O(5,21)}{O(5) \times O(21)} \rightarrow \frac{O(4,20)}{O(4) \times O(20)} \times \mathbb{R}^{+} \times \mathbb{R}^{24}
$$

corresponds to the weak (or strong) coupling limit of the type IIB string [5]; the $\mathbb{R}^{+}$factor corresponds to the type IIB string coupling constant, given by the exponential of the dilaton expectation value, while $\frac{O(4,20)}{O(4) \times O(20)}$ is the moduli space of conformal field theories on $K_{3}$ [42]. At weak coupling, the perturbative states correspond to the fundamental type IIB string, while at strong coupling we obtain the dual RR string, which is the fundamental string of the dual theory [9].

The decomposition

$$
\frac{O(5,21)}{O(5) \times O(21)} \rightarrow \frac{O(3,19)}{O(3) \times O(19)} \times \frac{S L(2, \mathbb{R})}{U(1)} \times \mathbb{R}^{+} \times \mathbb{R}^{45}
$$

appears to correspond to expanding in the volume of the $K_{3}$ surface $[5]: \frac{O(3,19)}{O(3) \times O(19)}$ is the moduli space of fixed volume $K_{3}$ metrics, $\frac{S L(2, R)}{U(1)}$ is the moduli space of type IIB string theories in $D=10$ and $\mathbb{R}^{+}$is the $K_{3}$ volume. However, the perturbative spectrum consists of two strings, which is unlike any conventional string theory. (The only way out would be if there were some metastable states dominating the perturbation theory, such as Kaluza-Klein modes on $K_{3}$. Note however that no such states should occur in the previous example of $n=1$ if the type IIB string interpretation is to hold.)

The perturbative spectra arising for $n>1$ are remarkable in that they are quite unlike anything that has been seen in the cases considered so far (again, unless some metastable states dominate). Recall that for a supergravity theory compactified on an $n$-torus, the perturbative spectrum for the coupling constant corresponding to the inverse volume of the torus consists of $n$ charged 0-brane multiplets that become massless supergravity multiplets in the large volume limit and which transform as an $\mathbf{n}$ under the $S L(n)$ torus mapping class group. Each of these $n$ multiplets carries a minimal charge $e_{0}$ with respect to a corresponding gauge 
field and has partners with charges $m e_{0}$ for all integers $m$, so that the spectrum includes $n$ Kaluza-Klein towers. The same perturbative spectrum emerged from superstring theories compactified on an $n$-torus, when expanded with respect to the inverse volume of the torus. Here we are finding something rather different: $n$ superstrings instead of $n$ super-0-branes. Furthermore, each of these has a charge $q_{0}$ with respect to a particular 2 -form $G^{+}$and has partners of charge $m q_{0}$ for all integers $m$, so that we are obtaining Kaluza-Klein-like towers of superstrings. These facts, together with the presence of the $S L(n, \mathbb{R}) / O(n)$ factor in (9.3) and comparison with the various limits of the non-chiral supergravities considered in the last section, suggest that the theory could have an interpretation in terms of a compactification of some theory on an $n$-torus (or perhaps on some space that is locally $T^{n}$, such as an orbifold of $T^{n}$ ). However, the required theory cannot be any known theory compactified on a torus in the conventional way from $n+6$ dimensions, as the wrong spectrum would emerge. For example, the case $n=5$ would require reduction from 11 dimensions. Reducing the 11-dimensional Mtheory on $T^{5}$ gives the usual type II string theory in $D=6$ which is rather different from what we want, although it is possible that reduction of M-theory on some orbifold of $T^{5}$ could work here.

To get more insight, consider the compactification of the chiral theory to 5dimensions on a circle or radius $R$, as this gives the 5-dimensional heterotic theory [3], with moduli space

$$
\frac{O(5,21)}{O(5) \times O(21)} \times \mathbb{R}^{+}
$$

Consider the coupling constant $g$ such that the limit $g \rightarrow \infty$ corresponds to the degeneration (9.3). Taking $g \rightarrow \infty$ corresponds to the decompactification to $5+n$ dimensions:- the heterotic string in 5-dimensions arises from the heterotic string in $5+n$ dimensions on compactification on a torus $T^{n}$ of volume $V$, and the coupling constant $g$ of the 5-dimensional theory corresponds to the volume $V$, so that $g \rightarrow \infty$ corresponds to $V \rightarrow \infty$. Thus taking $g \rightarrow \infty$, we recover Lorentz invariance in $5+n$ dimensions. On the other hand, taking $R \rightarrow \infty$ for fixed $g$ we recover 
6-dimensional Lorentz invariance. This suggests taking both decompactification limits together, $g \rightarrow \infty, R \rightarrow \infty$ might give a theory a $(6+n)$-dimensional theory, and in particular an 11-dimensional theory for $n=5$.

Unfortunately, the situation is not quite so simple as one has to be careful as to how the limits are taken. Consider the case $n=2$ for example. The string coupling constant $g_{7}$ of the $D=7$ heterotic string is given by $g_{7}^{2}=g^{3} R^{2}$, so that on taking the limit $g \rightarrow \infty$ one must take $R \rightarrow 0$ holding $g^{3} R^{2}$ constant if one is to obtain the heterotic string with finite coupling $g_{7}$. However, the heterotic string in $D=7$ is conjectured to be equivalent to M-theory compactified on a $K_{3}$ of volume $V=g_{7}^{4 / 3}$ [3], so that the limit $g_{7} \rightarrow \infty$ corresponds to the limit in which the volume of the $K_{3}$ becomes infinite, so that the theory decompactifies and 11-dimensional Lorentz invariance is regained. It is not clear whether this is related to the limit we are interested in here, which is given by first taking $R \rightarrow \infty$ to regain the type IIB string compactified on $K_{3}$ and then taking $g \rightarrow \infty$ so that the moduli space decomposes as in (9.6). However, it is plausible that the latter limit might define a theory in more than 6 dimensions.

Thus the strong coupling limit corresponding to (9.3) of the chiral $d=6$ theory, if it exists, appears to be a theory in at least six dimensions and probably more, which has an $n$-dimensional lattice of superstrings in the perturbative spectrum, acted on by $S L(n, \mathbb{Z})$. If, as suggested above, it arises from some theory compactified on $T^{n}$ in the limit of large torus volume, then the limiting theory should live in (at least) $6+n$-dimensions and should have a moduli space

$$
\frac{O(5-n, 21-n)}{O(5-n) \times O(21-n)}
$$

Note the absence of any dilaton-like $\mathbb{R}^{+}$factor. For $n=5$ there are two distinct limits (9.3) (cf [5]), so there could be two distinct theories in (at least) 11 dimensions in this case, and these would have no scalars and so no coupling constants and no perturbation theory. It will be convenient to refer to these theories as N-theories. There do not appear to be any known supergravity theories that could serve as 
the low-energy limits of these N-theories, so if these N-theories do exist, then either they do not have a low-energy effective field theory, or there are some new supergravity-type theories that are yet to be found.

So what could these N-theories be? One possibility is that they arise from orbifold compactifications of M-theory. Recall that the heterotic string in $10-m$ dimensions can be obtained by compactifying M-theory on $T^{m} \times S^{1} / \mathbb{Z}_{2}$ and the 5-dimensional heterotic string is equivalent to type IIB on $K_{3} \times S^{1}$ and hence to M-theory on $K_{3} \times T^{2}$. Moreover, $K_{3}$ can be obtained by blowing up the orbifold singularities of $T^{4} / \mathbb{Z}_{2}$. Then the N-theory corresponding to the degeneration (9.3) could arise from M-theory compactified on some orbifold of $T^{5-n}$, so that the $\mathrm{N}$ theory with $n=0$ - the chiral $D=6$ theory - would correspond to M-theory on some orbifold of $T^{5}$, and the degeneration (9.3) would correspond to a limit in the moduli space of the orbifold of $T^{5}=T^{n} \times T^{5-n}$ in which the volume of the $T^{n}$ became large*

Another intriguing possibility invokes a theory in twelve dimensions. Suppose that the N-theory corresponding to (9.3) lives in $d=6+n$ dimensions. When compactified on a circle, the N-theory in $d$ dimensions should give the heterotic string in $d-1$ dimensions. Indeed, the modulus for the circle would give an extra $\mathbb{R}^{+}$factor to (9.8), so that the correct heterotic string moduli space emerges. Consider for concreteness the $d=8 \mathrm{~N}$-theory which has moduli space

$$
\frac{O(3,19)}{O(3) \times O(19)}
$$

and which when compactified on $S^{1}$ should give the $d=7$ heterotic string, which is conjectured to be equivalent to M-theory on $K_{3}$ [3]. However, (9.9) is the moduli space for fixed-volume Ricci-flat metrics on $K_{3}$ and this suggests that $d=8 \mathrm{~N}$ theory might arise from some theory in 12 dimensions compactified on $K_{3}$. Let

\footnotetext{
$\star$ After this paper appeared, it was argued by Dasgupta and Mukhi and by Witten that the chiral theory in six dimensions given by compactifying the type IIB string on $K_{3}$ can also be obtained from M-theory by compactifying on the orbifold $T^{5} / \mathbb{Z}_{2}$.
} 
us suppose that there is such a theory in 12 dimensions - we shall refer to it as 'Y-theory' - and see what this would imply. The dynamics of Y-theory must be such that when compactified on $K_{3}$ the moduli space is not the space of all Ricci-flat metrics but only those of fixed volume, so that it is not a conventional theory of gravity. This could come about, for example, if Y-theory had some conformal invariance which led to the volume being a gauge degree of freedom, or if it was a theory of gravity subject to certain constraints, which removed the volume modulus. On further compactifying on $S^{1}$, one obtains the $D=7$ heterotic string, corresponding to M-theory compactified on $K_{3}$, and the radius of the circle provides the extra degree of freedom corresponding to the $K_{3}$ volume. This further suggests that Y-theory in $D=12$ can be compactified on $S^{1}$ in such a way that 'M-theory' emerges in $D=11$. Thus while compactifying from $D=11$ to $D=10$, the radius of the $S^{1}$ gives rise to the dilaton in $D=10$, the compactification from $D=12$ to $D=11$ gives rise to the extra gravitational degree of freedom that is needed to give unconstrained gravity in $D=11$.

This can be extended to the other degenerations. The degeneration (9.3) with $n \geq 2$ would correspond to Y-theory compactified on $\Xi^{6-n}$ and for $n \leq 2$ perhaps to $\mathrm{Y}$-theory on $K_{3} \times T^{2-n}$. Then a consistent picture seems to emerge which is similar to that proposed for the non-chiral $D=6$ theory and its limits in the previous section. It is conceivable that both the Y-theory picture and the orbifold picture are correct and that there is a relation between them, which would be similar to the relation between the Aspinwall-Morrison picture [5] and the HoravaWitten description [26] of the theories described in the last section. The type IIB theory might also be obtained from 12 dimensions, which would afford a geometrical interpretation of the $S L(2)$ symmetry, without needing to go through 9-dimensional theories, as in $[19,21]$. Needless to say, this is rather speculative, but if M-theory, why not Y-theory? 


\section{APPENDIX A}

The rescaling of the metric

$$
\tilde{g}_{\mu \nu}=e^{2 \Lambda} g_{\mu \nu}
$$

leads to the following changes in the curvature

$$
\tilde{R}=e^{-2 \Lambda}\left[R-2(D-1) \nabla^{2} \Lambda-(D-1)(D-2)(\nabla \Lambda)^{2}\right]
$$

$$
\sqrt{\tilde{g}} \tilde{R}=e^{(D-2) \Lambda} \sqrt{g}\left[R+(D-1)(D-2)(\nabla \Lambda)^{2}\right]-2 \sqrt{g}(D-1) \nabla^{\mu}\left(e^{(D-2) \Lambda} \nabla_{\mu} \Lambda\right)
$$

For an $n$-form potential $A_{n}=A_{\mu_{1} \mu_{2} \ldots \mu_{n}} d x^{\mu_{1}} d x^{\mu_{2}} \ldots d x^{\mu_{n}}$, the rescaling of the metric leads to

$$
\sqrt{g}\left|d A_{n}\right|^{2} \rightarrow \sqrt{g} e^{[D-2(n+1)] \Lambda}\left|d A_{n}\right|^{2}
$$

Acknowledgements: I would like to thank Michael Green, Ashoke Sen and Paul Townsend for valuable discussions. 


\section{REFERENCES}

1. C.M. Hull and P.K. Townsend, Nucl. Phys. B438 (1995) 109.

2. P.K. Townsend, Phys. Lett. B350 (1995) 184.

3. E. Witten, Nucl. Phys. B443 (1995) 85, hep-th/9503124.

4. C.M. Hull and P.K. Townsend, Nucl. Phys. B451 (1995) 525, hepth/9505073.

5. P.S. Aspinwall and D.R. Morrison, U-Duality and Integral Structures, hepth/9505025.

6. A. Sen, String-String Duality Conjecture in Six Dimensions and Charged Strings, hep-th/9504027.

7. J.A. Harvey and A. Strominger, The Heterotic String is a Soliton, hepth/9504047.

8. M.J. Duff, Strong/Weak Coupling Duality from the Dual String, hepth/9501030.

9. C.M.Hull, Phys. Lett. B357 (1995) 545, hep-th/9506194.

10. A. Dabholkar, Phys. Lett. B357 (1995) 307, hep-th/9506160.

11. A. Font, L. Ibanez, D. Lust and F. Quevedo, Phys. Lett. B249 (1990) 35;

S.J. Rey, Phys. Rev. D43 (1991) 526.

12. J.H. Schwarz and A. Sen, Nucl. Phys. B411 (1994) 35; Phys. Lett. 312B (1993) 105.

13. A. Sen, Nucl. Phys. B404 (1993) 109; Phys. Lett. 303B (1993); Int. J. Mod. Phys. A8 (1993) 5079; Mod. Phys. Lett. A8 (1993) 2023; Int. J. Mod. Phys. A9 (1994) 3707.

14. A. Ceresole, R. D'Auria, S. Ferrara and A. Van Proeyen, Nucl. Phys. B444 (1995) 92, hep-th/9502072.

15. A. Strominger, Nucl. Phys. B451, (1995) 96. 
16. C. Vafa, A stringy test of the fate of the conifold, hep-th/9505023.

17. B.R. Greene, D.R. Morrison and A. Strominger, Nucl. Phys. B451 (1995) 109, hep-th/9504145.

18. S. Kachru and C. Vafa, Nucl. Phys. B450 (1995) 69, hep-th/9505105.

19. E. Bergshoeff, C.M. Hull and T. Ortin, Nucl. Phys. B451 (1995) 547, hep-th/9504081.

20. P.K. Townsend, hep-th/9507048.

21. P.S. Aspinwall, hep-th/9508154.

22. J.H. Schwarz, hep-th/9508143, $9509148,9510086$.

23. J. Polchinski, preprint NSF-ITP-95-122, hep-th/951001.

24. E. Witten, preprint IASSNS-HEP-95-83, hep-th/9510135.

25. J. Polchinski and E. Witten, preprint IASSNS-HEP-95-81, hep-th/9510169.

26. P. Horava and E. Witten, preprint IASSNS-HEP-95-86 hep-th/9510209.

27. A. Dabholkar, G.W. Gibbons, J.A. Harvey and F. Ruiz-Ruiz, Nucl. Phys. B340 (1990) 33.

28. G.T. Horowitz and A. Strominger, Nucl. Phys. B360 (1991) 197.

29. C. Callan, J. Harvey and A. Strominger, Nucl. Phys. B359 (1991) 611.

30. M.J. Duff and J.X. Lu, Nucl. Phys. B354 (1991) 141; Phys. Rev. Lett. 66 (1991) 1402; Phys. Lett. 273B (1991) 409.

31. M.J. Duff, R.R. Khuri and J.X. Lu, Phys. Rep. 259 (1995) 213.

32. M.J. Duff, Class. Quant. Grav. 5 (1988) 189; A. Strominger, Nucl. Phys. B343 (1990) 167; M.J. Duff and J.X. Lu, Nucl. Phys. B354 (1991) 129 and Nucl. Phys. B357 (1991) 534.

33. M.B. Green and E. Witten, unpublished. 
34. E. Bergshoeff, M.B. Green, G. Papadopoulos and P.K. Townsend, hepth/9511079.

35. A.H. Chamsedine, hep-th/9510100.

36. E. Cremmer and B. Julia, Phys. Lett. 80B (1978) 48; Nucl. Phys. B159 (1979) 141.

37. S. Shenker, in Random Surfaces and Quantum Gravity, O. Alvarez, E. Marinari and P. Windey (eds.), Plenum (1991) 191.

38. G.W. Gibbons, M.B. Green and M.J. Perry, hep-th/9511080.

39. M.J. Duff and K.S. Stelle, Phys. Lett. 253B (1991) 113.

40. R. Güven, Phys. Lett. 276B (1992) 49.

41. P.K. Townsend, Phys. Lett. B354, (1995) 247, hep-th/9504095.

42. P.S. Aspinwall and D.R. Morrison, String Theory on K3 Surfaces, hepth/9404151, to appear in 'Essays on Mirror Manifolds 2'. 